\title{
SynCAMs Organize Synapses through Heterophilic Adhesion
}

\author{
Adam I. Fogel, ${ }^{1}$ Michael R. Akins, ${ }^{1 *}$ Alexander J. Krupp, ${ }^{2 *}$ Massimiliano Stagi, ${ }^{1 *}$ Valentin Stein,${ }^{2}$ and \\ Thomas Biederer ${ }^{1}$ \\ ${ }^{1}$ Department of Molecular Biophysics and Biochemistry, Yale University, New Haven, Connecticut 06520, and ${ }^{2}$ Max Planck Institute of Neurobiology, 82152 \\ Martinsried, Germany
}

Synapses are asymmetric cell junctions with precisely juxtaposed presynaptic and postsynaptic sides. Transsynaptic adhesion complexes are thought to organize developing synapses. The molecular composition of these complexes, however, remains incompletely understood, precluding us from understanding how adhesion across the synaptic cleft guides synapse development. Here, we define two immunoglobulin superfamily members, SynCAM 1 and 2, that are expressed in neurons in the developing brain and localize to excitatory and inhibitory synapses. They function as cell adhesion molecules and assemble with each other across the synaptic cleft into a specific, transsynaptic SynCAM 1/2 complex. Additionally, SynCAM 1 and 2 promote functional synapses as they increase the number of active presynaptic terminals and enhance excitatory neurotransmission. The interaction of SynCAM 1 and 2 is affected by glycosylation, indicating regulation of this adhesion complex by posttranslational modification. The SynCAM $1 / 2$ complex is representative for the highly defined adhesive patterns of this protein family, the four members of which are expressed in neurons in divergent expression profiles. SynCAMs 1, 2, and 3 each can bind themselves, yet preferentially assemble into specific, heterophilic complexes as shown for the synaptic SynCAM 1/2 interaction and a second complex comprising SynCAM 3 and 4. Our results define SynCAM proteins as components of novel heterophilic transsynaptic adhesion complexes that set up asymmetric interactions, with SynCAM proteins contributing to synapse organization and function.

Key words: SynCAM; CADM; synapse; synaptic adhesion; synaptogenesis; synaptic transmission

\section{Introduction}

Synapse organization in the CNS requires multiple specification steps to ensure synaptic function. These steps define nascent presynaptic and postsynaptic sites, support the distinct formation of excitatory or inhibitory synapses, and allow proper targeting of neurons. The development of synapses involves transsynaptic interactions of dedicated synaptic adhesion molecules (Scheiffele, 2003; Yamagata et al., 2003; Waites et al., 2005; Akins and Biederer, 2006). These adhesion systems likely comprise the protein complexes spanning the synaptic cleft (Lucic et al., 2005).

Despite the importance of synapse organization for brain development and function, our understanding of synaptic adhesion systems is limited. In both vertebrates and invertebrates, the diverse family of cadherin proteins is perhaps best understood to

\footnotetext{
Received June 16, 2007; revised Aug. 27, 2007; accepted Sept. 25, 2007.

This work was supported by National Institutes of Health Grants R01 DA018928 (T.B.) and P30 DA018343 (to the National Institute on Drug Abuse Neuroproteomics (enter, Yale University), March of Dimes Foundation Award 5-FY05-138 (T.B.), and The Brain Tumor Society (T.B.). We are grateful to Yuling Lei for excellent technical assistance, Nicholas Reish for technical support, Ewa Folta-Stogniew for performing light scattering experiments, and Clara L. Essmann and Julia Geiger for providing neurons for electrophysiological studies. We thank members of the Biederer laboratory and Drs. Michael R. Koelle, Anthony J. Koleske, and Sreeganga S. Chandra for helpful comments regarding this manuscript; Reinhard Jahn and Michaela Hellwig for synaptic vesicle fractionation samples; and Drs. Thomas $C$. Südhof, Eunjoon Kim, and Vance Lemmon for antibodies.

*M.R.A., A.J.K., and M.S. contributed equally to this work.

Correspondence should be addressed to Thomas Biederer, Department of Molecular Biophysics and Biochemistry, Yale University, 333 Cedar Street, New Haven, CT 06520. E-mail: thomas.biederer@yale.edu.

M. R. Akins's present address: Department of Neuroscience, Brown University, 185 Meeting Street, Providence, RI 02912 .

DOI:10.1523/JNEUROSCI.2739-07.2007

Copyright $\odot 2007$ Society for Neuroscience $\quad$ 0270-6474/07/2712516-15\$15.00/0
}

regulate synapses and neuronal signaling through transsynaptic interactions (Latefi and Colman, 2007; Takeichi, 2007). A different small family of synaptic adhesion molecules was identified in vertebrates with the purification of neurexins (Ushkaryov et al., 1992; Ushkaryov and Südhof, 1993) and their postsynaptic partners, neuroligins (Ichtchenko et al., 1995; Boucard et al., 2005). Neuroligins were shown to interact with neurexins to control the number of functional presynaptic terminals (Scheiffele et al., 2000; Dean et al., 2003; Levinson et al., 2005; Taniguchi et al., 2007). Conversely, neurexins cause postsynaptic protein assembly through binding of neuroligins (Graf et al., 2004; Chih et al., 2005). In vivo, neurexins and neuroligins affect synapse maturation, synaptic transmission, and network function (Missler et al., 2003; Varoqueaux et al., 2006).

In addition, various members of the Ig superfamily have been implicated as synapse organizing molecules. At the Drosophila neuromuscular junction, the adhesion molecule Fasciclin II stabilizes and patterns synapse formation (Davis et al., 1997). In the mollusk Aplysia, apCAM (Aplysia cell adhesion molecule) contributes to synaptic plasticity (Mayford et al., 1992), and in the nematode Caenorhabditis elegans, the epithelial protein synaptogenesis abnormal-2 (SYG-2) and the neuronal SYG-1 guide synapse specificity (Shen and Bargmann, 2003; Shen et al., 2004). In vertebrates, the Ig protein NCAM (neural cell adhesion molecule) regulates synapse formation and synaptic plasticity, whereas L1 participates in specifying synaptic sites, and the interactions of Sidekick family members contribute to synapse specification (Yamagata et al., 2003; Gerrow and El-Husseini, 2006).

Recently, the Ig superfamily member SynCAM 1 (synaptic cell 
adhesion molecule 1) was identified as synaptic cell adhesion molecule in vertebrates (Biederer et al., 2002). It is encoded by the gene CADM1 (cell adhesion molecule 1) and has also been named TSLC1, Necl-2 (nectin-like molecule 2), and RA175, reflecting its identification in other contexts (Kuramochi et al., 2001; Urase et al., 2001; Shingai et al., 2003). SynCAM 1 contains three extracellular Ig-like domains followed by a single transmembrane region and a short cytosolic sequence that interacts with PDZ [postsynaptic density-95 (PSD-95)/Discs large/zona occludens-1] domains of synaptic scaffolding molecules (Biederer et al., 2002). SynCAM 1 can engage in a homophilic interaction through its Ig-like domains to mediate cell adhesion (Biederer et al., 2002). In cultured hippocampal neurons, SynCAM 1 overexpression promotes excitatory neurotransmission. Furthermore, presentation of SynCAM 1 from non-neuronal cells to hippocampal neurons drives the neurons to develop fully functional excitatory presynaptic terminals at sites of contact (Biederer et al., 2002; Sara et al., 2005).

To advance our understanding of SynCAM 1 activity at synapses, we defined the transsynaptic adhesion complexes in which it participates. Guided by our recent analysis of the four SynCAM family members that share the same domain organization (Biederer, 2006), we considered them as candidate heterophilic binding partners of SynCAM 1. However, expression profiles and interaction patterns of the four SynCAM proteins as well as shared functions in brain have not been described. Specifically, it was not known whether they together confer synaptic adhesion and can contribute to synapse organization and function.

We here identify SynCAMs as a neuronal family of adhesion molecules that prefer heterophilic over homophilic interactions, enabling them to cooperate in synapse organization. SynCAM 1 and 2 assemble into a synaptic adhesion complex, and both proteins affect the organization and function of synapses. SynCAM 3 and 4 constitute the second strong heterophilic pair within the SynCAM family. The adhesion complex of SynCAM 1 and 2 is stable in synaptic membranes in vivo and its components recruit presynaptic proteins and promote excitatory neurotransmission in vitro. Together, our findings identify SynCAM proteins as components of an asymmetric transsynaptic adhesion system and introduce a novel molecular mechanism that can contribute to the diversity of transsynaptic interactions and guide synapse organization in the CNS.

\section{Materials and Methods}

Antibodies. Specific antibodies against SynCAM 1 (YUC8) were raised in chicken against the natively $\mathrm{N}$-deglycosylated extracellular sequence of mouse SynCAM 1 expressed as human IgG1 fusion in COS 7 cells. Antibodies against SynCAM 2 (YU524) and SynCAM 3 (YU525) were raised in rabbits against the peptides CKDVKYLKEEDANRKT and CHGDQTRIQEDPNGKT, respectively, corresponding to amino acid sequences in the second Ig-like domain of the mouse proteins with an $\mathrm{N}$-terminal cysteine for coupling. Antibodies against SynCAM 4 (YU591) were raised in rabbits against the peptide CGGDGHKRKEEFFI, corresponding to the $\mathrm{C}$-terminal sequence of the mouse protein with an $\mathrm{N}$-terminal cysteine for coupling. All polyclonal antibodies were affinity purified using standard procedures (Harlow and Lane, 1999). For simultaneous detection of SynCAM 1-3, we used a pleioSynCAM antibody (T2412) raised in rabbits against the SynCAM 1 C-terminal sequence described previously (Biederer et al., 2002) that also recognizes this conserved sequence in SynCAM 2 and 3, but not 4 (supplemental Fig. 1, available at www.jneurosci.org as supplemental material). For immunoprecipitation of SynCAM 1 and selected immunoblots as described in the figures, monoclonal antibodies raised in chicken against its extracellular domain were used (clone 3E1; MBL Laboratories, Nagoya, Japan) (supplemental Fig. 1, available at www.jneurosci.org as supplemental mate- rial). Antibodies to synaptotagmin 1 (for immunoblotting, 41.1; for synaptic vesicle uptake, 604.1), synaptophysin (7.2), synapsin (1006002/ E028), gephyrin (147011), and GDP dissociation inhibitor (GDI) (81.2) were obtained from Synaptic Systems (Göttingen, Germany); to PSD-95 (MA1-045) from Affinity Bioreagents (Golden, CO); to N-cadherin from BD Biosciences (San Jose, CA); to flag (M2) from Sigma (St. Louis, $\mathrm{MO}$ ); and to NeuN (neuronal-specific nuclear protein) (MAB377), vGlut1 (AB5905), vGlut2 (AB5907), and GAD65 (AB5082) from Millipore (Billerica, MA). Monoclonal antibodies to actin (JLA20; developed by Jim Jung-Ching Lin, University of Iowa, Iowa City, IA) and SV2 (developed by Kathleen Buckley, Harvard Medical School, Boston, MA) were obtained from the Developmental Studies Hybridoma Bank maintained by the University of Iowa (Iowa City, IA). Antibodies to valosincontaining protein (VCP) (Sugita and Sudhof, 2000) were a gift from Dr. Thomas Südhof (University of Texas Southwestern Medical Center, Dallas, TX); antibodies to Netrin-G ligand (NGL) (Kim et al., 2006) were a gift from Dr. Eunjoon Kim (KAIST, Republic of South Korea); and antibodies to L1 were a gift from Dr. Vance Lemmon (University of Miami, Miami, FL).

Vector construction and heterologous protein expression. Sequences encoding full-length SynCAM 2 (splice product 2) (Biederer, 2006), SynCAM 3 (splice product 2), and SynCAM 4 were amplified from a mouse brain cDNA library prepared at 9-11 weeks of age (Clontech \#639400). PCR products were subcloned into the eukaryotic expression vector pCMV5 for heterologous expression or for long-lasting expression in neurons into the vector pCAGGS (a gift from Dr. Jun-ichi Miyazaki, Osaka University, Osaka, Japan) (Niwa et al., 1991).

To obtain intracellularly tagged SynCAM constructs for heterologous expression, NheI sites were introduced in full-length SynCAM sequences using the PCR mutagenesis kit (Stratagene, La Jolla, CA) to generate pCMV5 expression vectors for SynCAM1 $(420)^{*}$ NheI, SynCAM2 $(391)^{*}$ NheI, SynCAM3 (377) ${ }^{\star}$ NheI, and SynCAM4 $(369)^{\star}$ NheI, with bracketed numbers indicating the amino acid into the codon of which the restriction site was introduced. A single flag epitope was inserted into the NheI site using annealed oligos. To introduce monomeric cyan fluorescent protein (CFP), its sequence was PCR amplified from pRSET-B mCFP (a gift from Dr. Roger Tsien, University of California San Diego, San Diego, CA) and subcloned using NheI into the pCMV5-SynCAM ${ }^{\star} N h e I$ expression vectors. Proper sorting of tagged proteins was confirmed by surface biotinylation (data not shown). To generate SynCAM 1 and 2 constructs with an extracellular flag epitope for expression in neurons, the vectors pCAGGSSynCAM1 $(363)^{*}$ NheI and pCAGGS-SynCAM2(326)*NheI were generated by PCR mutagenesis, and flag epitopes were inserted as described above. Bracketed numbers indicate the amino acid into the codon of which the restriction site was introduced.

To obtain expression vectors for the full extracellular sequences of SynCAM 2-4 fused to human IgG1, these sequences were PCR amplified and subcloned into pCMVIG9. pCMVIG9-SynCAM1 extracellular domain (ECD) has been described previously (Biederer et al., 2002). To obtain pCMVIG9 expression vectors for individual or combined Ig domains of the SynCAM 1 ECD, these sequences were PCR amplified and subcloned into pCVMIG9 or pCVMIG9-SynCAM1 $\triangle$ IgECD (Biederer et al., 2002). To express extracellular SynCAM sequences with a thrombin cleavage site $\mathrm{N}$-terminal of an IgG1 sequence, sequences were amplified by PCR and subcloned into the pDT100 vector (a gift from Dr. Dimitar Nikolov, Sloan-Kettering Institute, New York, NY).

To generate vectors for Semliki forest viral particle production and expression in neurons, sequences encoding wild-type, full-length SynCAM 1 and 2 were PCR amplified and subcloned into pSCA (DiCiommo and Bremner, 1998).

For heterologous expression in human embryonic kidney-293 (HEK 293) cells, cells were transfected with FuGENE 6 (Roche Applied Science, Indianapolis, IN). COS7 cells were transfected by the DEAE-dextran method (Gorman, 1985), and IgG1 fusion proteins were purified as described previously (Sugita et al., 2001).

In situ hybridization. For chromogenic detection, free-floating $40 \mu \mathrm{m}$ sections were incubated in the appropriate riboprobe at $1 \mathrm{ng} / \mu \mathrm{l}$ in hybridization solution overnight at $65^{\circ} \mathrm{C}$ following standard procedures. Sections were then incubated in nitroblue-tetrazolium-chloride/5- 
bromo-4-chlor-indolyl-phosphate (Roche Applied Science) and mounted. For fluorescence detection, $20 \mu \mathrm{m}$ sections were slidemounted and incubated with riboprobes as described for the chromogenic detection. Tissue was then incubated in block [ $1 \%$ blocking reagent (Roche Applied Science) in TBS and 0.1 Triton X-100 (TBST)] containing a mouse anti-NeuN antibody to detect neuronal nuclei (Mullen et al., 1992) (1:300; Millipore MAB377), followed by incubation with a polyclonal antibody against digoxigenin conjugated to alkaline phosphatase (Roche Applied Sciences) in block. Sections were rinsed, incubated in block containing anti-mouse IgG1 conjugated to Alexa 488 (1:1000; Invitrogen, San Diego, CA) to detect NeuN staining, then incubated in HNPP/Fast Red (Roche Applied Sciences) to detect the riboprobes, rinsed, and mounted. Riboprobe and protocol details are available on request. Digital images were collected using a Microtek (Torrance, CA) Scanmaker 1900 scanner (chromogen images) or collected using a Hamamatsu (Bridgewater, NJ) Orca camera attached to a Nikon (Tokyo, Japan) Eclipse TE2000-U microscope (fluorescence images). Signal level coding was generated using NIH ImageJ. The Lookup Table was switched to 16-color, and signal quantitation was performed using the ImageJ macro "BackgroundCorrectedDensity." The signal for each region was then normalized to the total signal in the hippocampus and divided by the area of that region.

Brain fractionation and glycosylation analysis. Tissue samples were prepared from rats by rapid homogenization. Rat brain homogenates were subfractionated by the method of Jones and Matus (1974) with modifications (Biederer et al., 2002). Preparation of highly purified synaptic vesicles was performed as described previously (Takamori et al., 2006). Enzymatic glycosylation analysis was performed using sialidase (neuraminidase; Roche Applied Science) and PNGase F (N-glycosidase F; New England Biolabs, Beverly, MA) according to the manufacturer instructions. Carbohydrate contents of SynCAM extracellular domains were determined by size exclusion chromatography/laser static light scattering (SEC/LS) analysis (Hayashi et al., 1989). Analyzed proteins were expressed from $\mathrm{pDT}$ vectors in COS7 cells, and the IgG1 fusion protein was cleaved off with thrombin (Roche Applied Science). Amounts of purified SynCAM 4 were too low for SEC/LS analysis.

Interaction analyses. Bead clustering was performed using $1 \mu \mathrm{m}$ diameter FluoSpheres (Invitrogen) after covalent coupling of Protein A to the beads using 1-ethyl-3-(3-dimethylaminopropyl)-carbodiimide (Invitrogen). SynCAM extracellular domain-IgG1 fusion proteins or control IgG were bound to Protein A-FluoSphere beads of red or green color, respectively. After agitation at $4^{\circ} \mathrm{C}$ for $1 \mathrm{~h}$ in 24 -well plates, suspensions were imaged with a Hamamatsu Orca ER camera attached to a Nikon Eclipse TE2000-U. Bead clustering was not used for studies of heterophilic SynCAM binding, because homophilically clustered beads could not be sufficiently dispersed to allow heterophilic mixing.

For affinity chromatography on SynCAM extracellular domains, rat forebrain homogenate was prepared in homogenization buffer $(10 \mathrm{mM}$ HEPES-KOH, pH 7.4, $25 \mathrm{~mm}$ potassium acetate, $320 \mathrm{~mm}$ sucrose) in the presence of protease inhibitors $[1 \mathrm{mg} / \mathrm{l}$ pepstatin, $1 \mathrm{mg} / \mathrm{l}$ aprotinin, 10 mg/l leupeptin (all from Roche Applied Science), 0.5 mm PMSF (Sigma)], centrifuged to obtain the postnuclear supernatant, and membranes were pelleted in a Beckman Ti 70 (Beckman Instruments, Fullerton, CA $)$ at $60,000 \times g(k$-factor 59,1285$)$ for $30 \mathrm{~min}$ at $2^{\circ} \mathrm{C}$. Membrane proteins were solubilized in homogenization buffer containing $1.0 \%$ 3-[(3-cholamidopropyl)dimethylammonio]-1-propanesulfonate (Roche Applied Science), and precleared on Protein A agarose (Invitrogen). As affinity matrix, SynCAM extracellular domains heterologously expressed in COS7 cells were bound to Protein A agarose beads and covalently coupled with dimethyl pimelimidate (Sigma). Solubilisates were then loaded onto these beads, and bound proteins were sequentially eluted with $800 \mathrm{~mm}$ potassium acetate followed by $2 \%$ SDS. For previous native deglycosylation of the immobilized SynCAM extracellular domain, PNGase F (New England Biolabs) was used. Immunoprecipitation of SynCAM 1 complexes was performed from crude synaptosomes prepared from forebrains of postnatal day 15 (P15) or adult rats that were solubilized with Triton X-100 (Roche Applied Science) and subjected to immunoprecipitation with a monoclonal anti-SynCAM 1 antibody
(MBL Laboratories, 3E1; 1:200). Analysis was performed by immunoblotting.

For homophilic cell adhesion analysis, HEK 293 cells individually expressing intracellularly CFP-tagged SynCAMs were cultured and analyzed by fluorescence microscopy. For analysis of heterophilic adhesion, cells expressing either intracellularly flag-tagged SynCAM 1 or CFPepitope tagged SynCAMs were mixed at a 1:1 ratio, plated together, and analyzed after immunostaining with anti-flag antibodies by fluorescence microscopy.

Immunocytochemistry and mixed cocultures. Fluorescence images for adhesion analysis in heterologously expressing cells were acquired after immunostaining with anti-flag antibodies on a Nikon Eclipse TE-U 2000 with an attached Hamamatsu Orca ER camera.

For localization of SynCAM 1 and 2 in neurons, dissociated cultures of hippocampal neurons were prepared at $\mathrm{P} 0$ or $\mathrm{Pl}$ as described previously (Biederer and Scheiffele, 2007) and transfected at $7 \mathrm{~d}$ in vitro (d.i.v.) with pCAGGS vectors encoding extracellularly flag-epitope-tagged SynCAM proteins using Lipofectamine LTX (Invitrogen). Mature neuronal cultures were analyzed at 21 d.i.v. after immunostaining with antibodies directed against the synaptic marker synapsin (1:500) and the flag epitope (1:500) to visualize tagged SynCAM proteins, or against SV2 (1:500) and pleioSynCAM antibodies (T2412, 1:500) to visualize endogenous SynCAM proteins. Excitatory synaptic specializations were visualized with anti-vGlut1/2 antibodies (each 1:2000; applied in combination) and anti-PSD-95 antibodies (1:500). Inhibitory synaptic

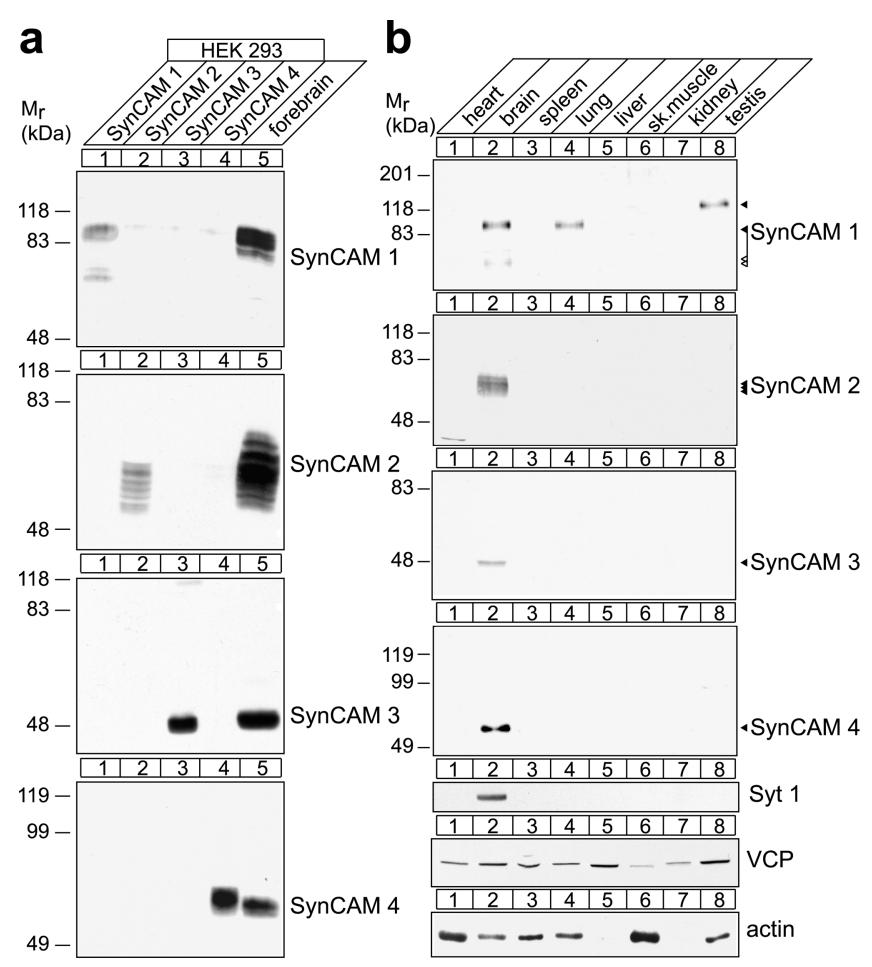

Figure 1. SynCAM proteins are prominently expressed in brain. $\boldsymbol{a}$, Specificity of antibodies raised for this study. HEK 293 cells were transfected with expression constructs encoding fulllength mouse SynCAM 1-4. Total cell lysates were prepared, and equal lysate fractions were analyzed by immunoblotting (lanes $1-4$ ). Twenty micrograms of adult rat forebrain proteins were analyzed as positive control (lane 5). Top to bottom, Immunoblots probed with antibodies raised against the purified, natively N-deglycosylated full-length SynCAM 1 extracellular domain (YUC8, raised in chicken), against an extracellular SynCAM 2 peptide (YU524, raised in rabbit), against an extracellular SynCAM 3 peptide (YU525, raised in rabbit), and against an intracellular SynCAM 4 peptide (YU591, raised in rabbit). The numbers on the left indicate positions of molecular weight markers, and arrowheads show the running positions of the indicated SynCAM proteins. $\boldsymbol{b}$, Tissue profile of SynCAM expression. The indicated tissues were prepared from adult rats, and equal protein amounts were analyzed by immunoblotting for the proteins shown. Synaptotagmin 1 (Syt 1) served as brain-specific control, and the widely expressed VCP and actin were additional loading controls. 


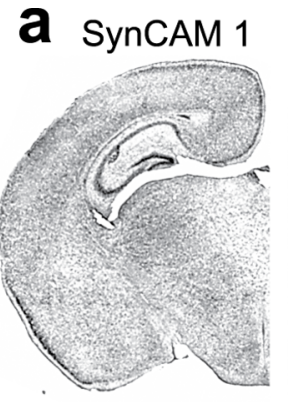

SynCAM 2

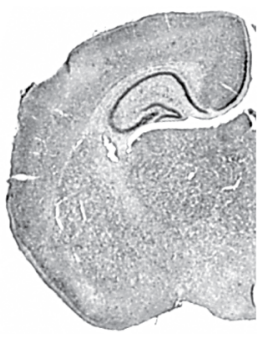

SynCAM 2
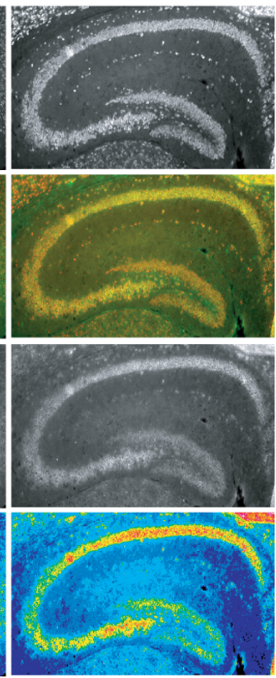

C

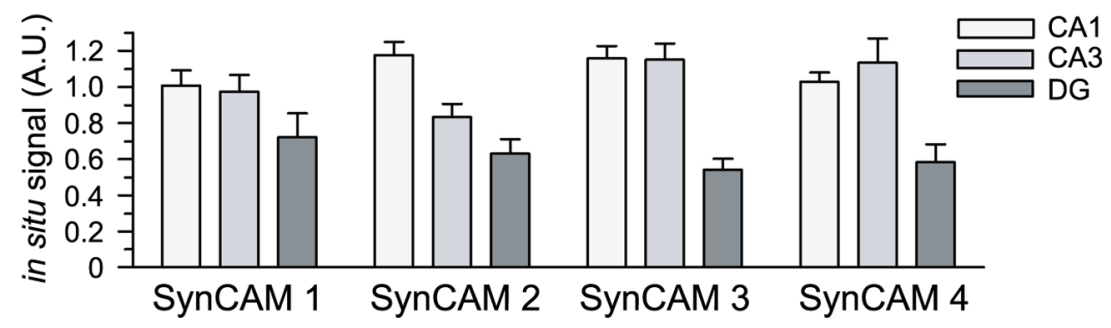

Figure 2. SynCAM proteins are predominantly expressed in neurons and display differential expression patterns in the hippocampus. $\boldsymbol{a}$, SynCAM family members are broadly expressed in forebrain regions. Coronal sections of mouse brain at P15 were analyzed by in situ hybridization. No region was seen that lacked SynCAM staining. Scale bar, $1 \mathrm{~mm}$. $\boldsymbol{b}$, SynCAMs are expressed by neurons in the hippocampus at P15 in overlapping but distinct patterns. Immunostaining for the neuronal nuclear marker NeuN (top row) was performed in parallel with in situ hybridization for SynCAMs (third row). The merged images show that most SynCAM-expressing cells are positive for NeuN (second row; NeuN staining, red; SynCAM hybridization signal, green). Pyramidal cells of the CA fields and granule cells of the dentate gyrus differed in SynCAM expression as visualized by pseudocolor rendering of the in situ hybridization signals (bottom row). The color code shown represents signal intensities (blue, lowest signal; red, highest). SynCAM 1 displayed uniform expression across the three examined regions, whereas SynCAM 2 is more prominent in the CA1 field. SynCAM 3 and 4 appear enriched in CA1 and CA3 fields relative to dentate gyrus. Results are quantitated in $c$. Images correspond to typical results from four hippocampi of two mice. c, Quantification of regional SynCAM expression differences in P15 hippocampus. The pyramidal cell layers of the CA fields and granule cell layer of the dentate gyrus were outlined, and the fluorescent in situ hybridization signal in each hippocampal region was normalized to the total signal and to the area of the individual region. Bar graphs show average SynCAM transcript hybridization signals \pm SEM for four hippocampi from two animals analyzed by in situ hybridization. A.U., Arbitrary units.

specializations were detected with anti-GAD65 antibodies (1:300) and anti-gephyrin antibodies (1:500). Images were acquired on a Zeiss (Oberkochen, Germany) LSM 510 META laser scanning confocal microscope, with channels scanned separately to avoid signal contagion and a pinhole set to $1 \mu \mathrm{m}$ for each channel. Images were quantitatively analyzed using Matlab (MathWorks, Natick, MA). Briefly, the script is based on the functions [graythresh], [bwareaopen], and [label2rgb] present in the Matlab Image Toolbox. The script is available on request.
Mixed coculture experiments of dissociated hippocampal neurons with HEK 293 cells expressing SynCAM proteins were performed with modifications as described previously (Biederer and Scheiffele, 2007). Briefly, HEK 293 cells expressing soluble CFP or intracellularly CFP-tagged SynCAM 1 or 2 were seeded atop neurons at 9 d.i.v. The activity analysis of extracellularly flag-tagged SynCAMs used their cotransfection with soluble CFP into HEK 293 cells to define transfected cells. Immunostaining of mixed cocultures for the presynaptic marker synapsin was performed at 11 d.i.v., and images were acquired by confocal microscopy as described above. We used Matlab (MathWorks) to quantify the surface area fraction of HEK 293 cells that is immunopositive for synapsin. Briefly, the script uses the function [graythresh] to threshold the first channel with the fluorescence signal from a given HEK 293 cell. This defines the perimeter of the cell as mask for the second channel with the fluorescence signal of the analyzed synaptic marker. Every image was acquired with identical settings on the microscope and analyzed with the same thresholds. The output was then calculated by dividing the surface area of channel two by the surface area of the masked and thresholded channel one. The script is available on request. Images were collected blind to the synaptic marker channel.

To modify this mixed coculture assay for recruitment studies, neurons were transfected at 7 d.i.v. with a pCAGGS expression vector encoding extracellularly flag-tagged SynCAM 1 or 2 using Lipofectamine LTX. HEK 293 cells expressing CFP-tagged SynCAM 2 or 1, respectively, were seeded atop these neurons at 9 d.i.v. Immunostaining for the flag epitope and synapsin was performed at 11 d.i.v., and images were acquired by confocal microscopy as described above.

Physiological studies. To examine effects of postsynaptic SynCAM overexpression on presynaptic vesicle recycling, dissociated hippocampal neurons were prepared (Biederer and Scheiffele, 2007) and cotransfected at 7 d.i.v. with pCAGGS vectors encoding SynCAM proteins and soluble green fluorescent protein (GFP), or transfected with GFP alone as negative control. At 11 d.i.v., presynaptic terminals were labeled under depolarizing conditions by uptake of antibodies against the luminal domain of synaptotagmin 1 (Synaptic Systems; Cl 604.1) as described previously (Biederer and Scheiffele, 2007). After staining with appropriate secondary antibodies against synaptotagmin 1, GFP-positive neurons displaying pyramidal morphology were selected for analysis. Images of proximal dendrites were obtained using a Nikon Eclipse TE-U 2000 fluorescence microscope with an attached Hamamatsu Orca ER camera. Area and density per proximal dendrite length of synaptotagmin 1-positive puncta were determined using IPLab software (BD Biosciences Bioimaging). Analyses were performed blinded to experimental condition.

Electrophysiological analysis was performed in primary hippocampal cultures prepared from embryonic day 18 (E18) to E19 Sprague Dawley rats. Neurons were grown in Neurobasal B27 medium for 7-9 d.i.v. and 


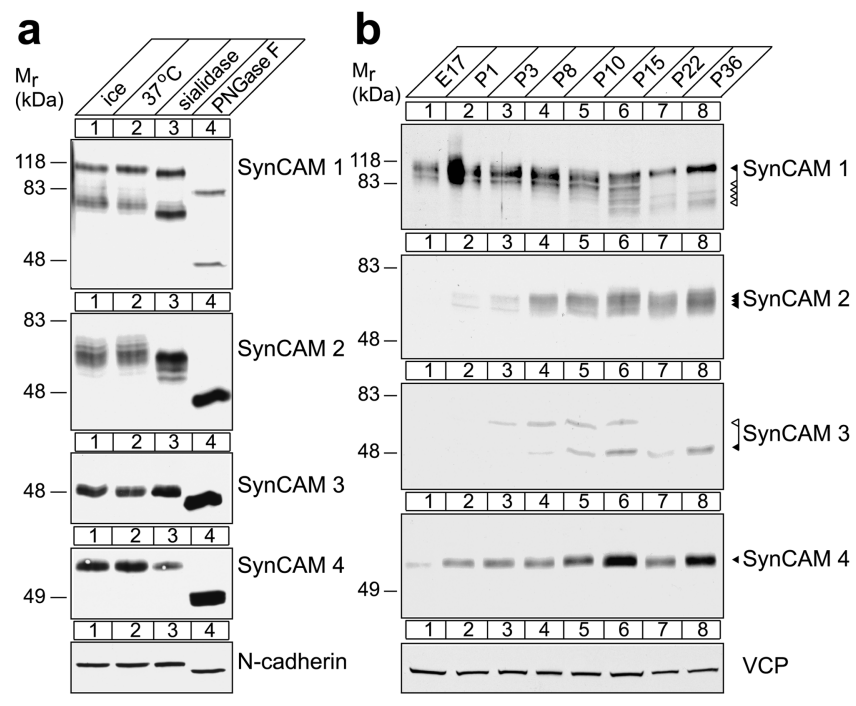

Figure 3. SynCAM proteins are distinctly glycosylated and prominently expressed in brain during early postnatal development. $\boldsymbol{a}$, SynCAM proteins are differentially glycosylated. A membrane fraction was prepared from adult rat forebrain, and equal protein amounts were subjected to enzymatic deglycosylation at $37^{\circ} \mathrm{C}$ with sialidase to remove sialic acids or PNGase $F$ to remove $\mathrm{N}$-linked carbohydrates. Control samples were incubated in parallel as indicated. In each lane, $30 \mu \mathrm{g}$ of total brain protein was loaded. Immunoblots were probed with antibodies against SynCAM 1- 4 or the N-glycosylated control protein N-cadherin as indicated. The numbers on the left indicate positions of molecular weight markers. $\boldsymbol{b}$, Developmental expression profile of SynCAM proteins in brain. Total rat brain proteins were prepared at the indicated embryonic $(E)$ and postnatal $(P)$ days. Thirty micrograms of protein were analyzed per lane by immunoblotting for expression of SynCAM 1-4. VCP served as loading control. The numbers on the left indicate positions of molecular weight markers. Arrowheads show the running positions of the indicated SynCAM proteins in their predominantly adult (black) and postnatal (white) glycosylation forms.

transduced using the Semliki forest virus system to coexpress full-length, wild-type SynCAM proteins and soluble GFP. Twelve to $24 \mathrm{~h}$ posttransduction, patch-clamp recordings of $\mathrm{mEPSC}$ were performed from transduced neurons identified by their GFP expression in the presence of 200 $\mathrm{nm}$ tetrodotoxin and $100 \mu \mathrm{M}$ picrotoxin. Protocol details are available on request. Analyses were performed blinded to experimental condition.

Miscellaneous procedures. Protein concentrations were determined using the Pierce BCA assay. SDS-PAGE and immunoblotting were performed using standard procedures. All animal procedures undertaken in this study were approved by the Yale University Institutional Animal Care and Use Committee and were in compliance with National Institutes of Health guidelines (for T.B.) and governmental regulations by the Regierung von Oberbayern (for V.S.).

\section{Results}

The four SynCAMs are predominantly and distinctly expressed in neurons

To characterize the four SynCAM proteins, we raised specific antibodies against each of them (Fig. 1a). Immunoblot analysis revealed that SynCAM tissue expression is highly restricted (Fig. $1 b)$. SynCAM 1 is most prominently expressed in brain and also found in lung and testis. SynCAM 2, 3, and 4 are expressed exclusively in brain among the tissues analyzed. The expression pattern of SynCAM 1 is in agreement with its known roles as synaptic adhesion molecule within the CNS (Biederer et al., 2002; Sara et al., 2005) and outside the CNS as tumor suppressor of non-small-cell lung cancer (Murakami, 2005) and as mediator of spermatid maturation (Fujita et al., 2006). The predominant expression of SynCAM proteins in brain, but not other tissues, is unusual among Ig superfamily members and indicates brainspecific functions.
In which brain regions are SynCAMs expressed, and do they function in neurons? The antibodies we raised were not applicable for comparative immunohistochemical detection of the four SynCAM proteins. We therefore performed in situ hybridization studies to localize SynCAM transcripts at P15 and address these questions within the peak period of synaptogenesis (Harris et al., 1992; Fiala et al., 1998). Each SynCAM is broadly expressed in forebrain, with some expression found in all brain areas examined, indicating ubiquitous functions in brain (Fig. 2a). Analysis of hippocampal sections by immunostaining for the neuronal nuclear marker NeuN combined with fluorescent in situ hybridization for SynCAMs demonstrated that all four family members are expressed predominantly in neurons at P15 (Fig. $2 b$ ). The vast majority of cells were positive both for NeuN and SynCAM transcripts, consistent with most SynCAM-expressing cells being neurons at this developmental time point. Few SynCAM-positive cells lacked NeuN staining and could be non-neuronal. We noted that neuronal expression of SynCAMs in hippocampus is not uniform. SynCAMs were differentially transcribed by the pyramidal cells of the CA fields and the granule cells of the dentate gyrus as visualized by pseudocolor rendering of the in situ hybridization signals (Fig. 2b, bottom row). Quantification of the in situ hybridization signals indicated that SynCAM 1 was evenly expressed across these hippocampal regions, whereas SynCAM 2 appeared most strongly expressed in the CA1 field (Fig. 2c). SynCAM 3 and 4 appeared enriched in both CA fields relative to the dentate gyrus. SynCAMs are therefore likely expressed in distinct ratios between different neuronal populations, contributing to the diverse surface expression patterns of neurons.

\section{SynCAM Ig-like domains are differentially glycosylated during brain development}

The open reading frames of the four mouse SynCAM genes encode proteins of 40.2-45.1 kDa (Biederer, 2006). However, SynCAM proteins have higher apparent molecular weights in the adult rodent forebrain (SynCAM 1, $100 \mathrm{kDa}$; SynCAM 2, 62-76 kDa; SynCAM 3 , $49 \mathrm{kDa}$; SynCAM 4, $67 \mathrm{kDa}$ ) (Fig. 1a, lane 5). All SynCAMs contain multiple predicted $\mathrm{N}$-glycosylation sites in their extracellular Ig-like domains (Biederer, 2006) that create these distinct molecular weight differences within and between SynCAM family members as shown by enzymatic deglycosylation of brain samples (Fig. 3a). Here, SynCAM 1 and 2 exist as heavily glycosylated and diverse protein species distinguished by the high amount of $\mathrm{N}$-linked carbohydrates and sialic acids they carry. In contrast, SynCAM 3 and 4 carry less $\mathrm{N}$-linked carbohydrates, are molecularly less diverse, and display no detectable sialic acids. After complete N-deglycosylation with PNGase F, a fraction of SynCAM 1 and all other SynCAMs are detected at the approximate molecular weights predicted by their open reading frames. O-glycosylation is not removed by these enzymatic treatments and likely accounts for the remaining SynCAM 1 population that persists at an apparent molecular weight at $\sim 75 \mathrm{kDa}$ after $\mathrm{N}$-deglycosylation. Alternative splicing allows for the expression of corresponding SynCAM 1 isoforms, which can contain either none or up to 21 predicted O-glycosylation sites (Biederer, 2006).

SynCAM proteins expressed in HEK 293 cells are glycosylated similar as in adult brain (Fig. 1a). We therefore determined the carbohydrate content of their heterologously expressed extracellular domains by light scattering analysis. Consistent with our enzymatic deglycosylation results of brain SynCAMs and their apparent molecular weights, SynCAM 1 and 2 are extensively glycosylated, carrying $0.53 \mathrm{~g}$ of sugar/gram of protein and $0.15 \mathrm{~g}$ of sugar/gram of protein, respectively, whereas SynCAM 3 contains only $0.04 \mathrm{~g}$ of sugar/gram of protein. This establishes the 


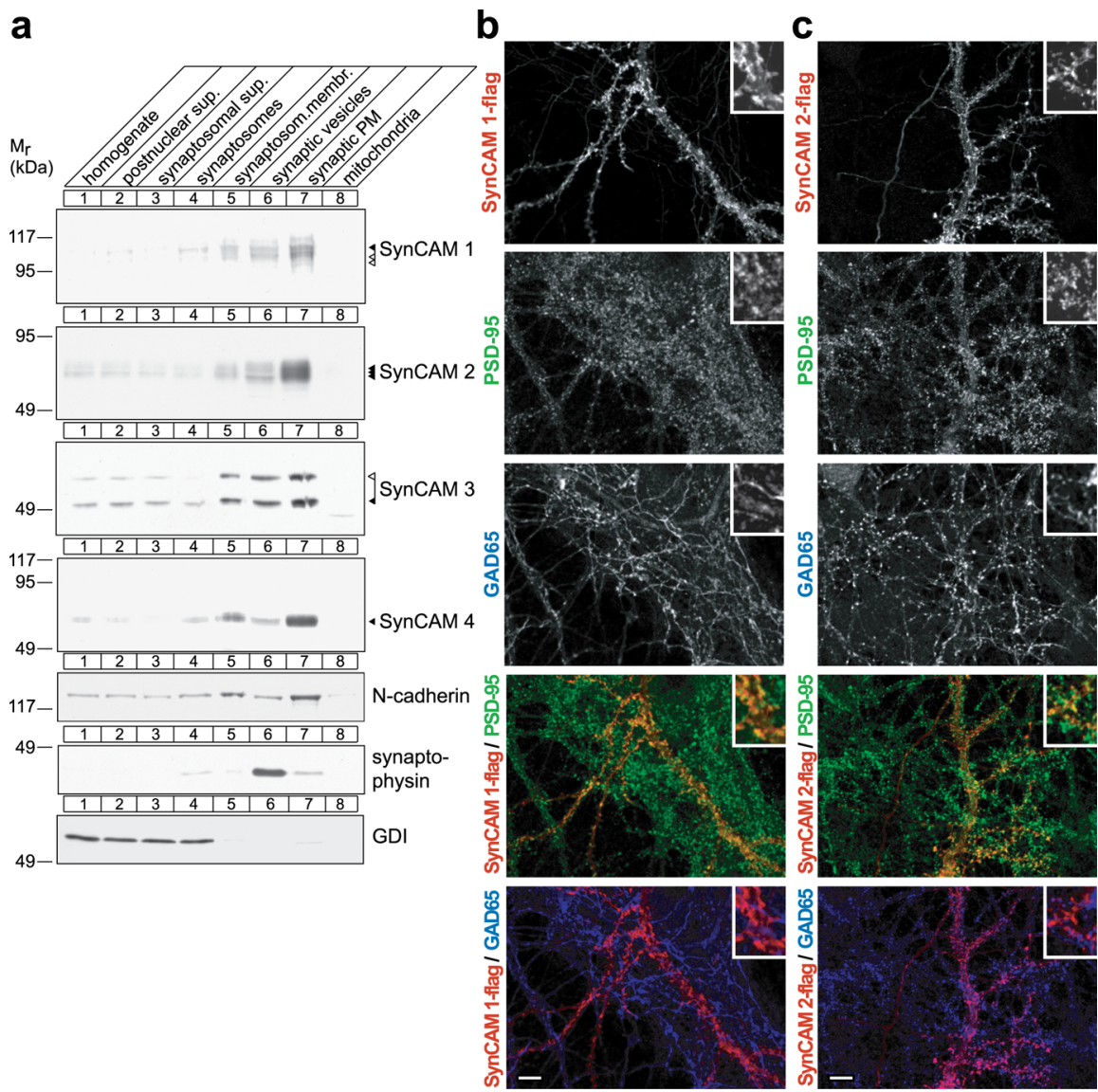

Figure 4. SynCAMs are synaptic plasma membrane proteins present at excitatory but also found at inhibitory synaptic specializations. $\boldsymbol{a}$, Synaptic plasma membrane fractionation of SynCAM proteins. The indicated subcellular fractions were prepared from rat forebrain at P9. Thirty micrograms of each fraction were analyzed by immunoblotting for the four SynCAM proteins as indicated. The synaptic membrane protein N-cadherin, the synaptic vesicle protein synaptophysin, and the soluble protein GD were markers for these respective fractions. The numbers on the left indicate positions of molecular weight markers. Arrowheads show the running positions of the indicated SynCAM proteins in their predominantly adult (black) and postnatal (white) glycosylation forms. $\boldsymbol{b}$, Exogenously expressed SynCAM 1 is sorted to both excitatory and inhibitory synaptic specializations in dissociated hippocampal neurons. Epitope-tagged SynCAM 1-flag was transfected into dissociated hippocampal neurons at 7 d.i.v. After transfection, mature cultures were analyzed at 21 d.i.v. by confocal microscopy after triple immunostaining for flag, the inhibitory presynaptic vesicle marker GAD65, and the postsynaptic excitatory marker PSD-95. Individual immunostainings are shown in the gray scale panels as indicated. The two panels at the bottom depict the indicated double merged images (SynCAM 1-flag, red; PSD-95, green; GAD65, blue). Insets in the top right of each panel represent 10-fold enlarged areas of the image shown. Confoca images were analyzed using Matlab for the number of single puncta and the occurrence of their double colocalization (SynCAM 1-flag, 2461 puncta analyzed; 3 images). Double colocalization identifies $53 \pm 23 \%$ of SynCAM 1-flag puncta as immunopositive for PSD-95, and $24 \pm 12 \%$ of SynCAM 1-flag puncta as immunopositive for GAD65. Errors are stated as SDs. The remaining SynCAM 1-flag puncta are removed from the synaptic markers analyzed here and nonsynaptic. We observed an occasional colocalization of GAD65 and PSD-95 in our hippocampal cultures, quantitated as an $\sim 10 \%$ mismatch of excitatory and inhibitory synaptic specializations similarly as described previously (Anderson et al., 2004). Scale bar, $10 \mu \mathrm{m}$. c, SynCAM 2 colocalizes with excitatory and inhibitory synapse markers in cultured hippocampal neurons. Epitope-tagged SynCAM 2-flag was expressed and analyzed as described in $\boldsymbol{a}$ (SynCAM 2-flag, 1951 puncta analyzed; 4 images). Double colocalization identifies $62 \pm 28 \%$ of SynCAM 2-flag puncta as immunopositive for PSD-95, and $31 \pm 12 \%$ of SynCAM 2-flag puncta as immunopositive for GAD65. Scale bar, $10 \mu \mathrm{m}$. The immunostainings shown in $\boldsymbol{b}$ and $\boldsymbol{c}$ are representative of the quantification results.

four SynCAM proteins as complex glycoproteins primarily expressed in brain.

To guide our understanding of SynCAM function in brain development, we determined their protein expression profile in the rat brain (Fig. 3b). SynCAM 1 and 4 are already expressed in late embryonic stages, whereas SynCAM 2 and 3 are first expressed postnatally around P3. All SynCAMs are prominently expressed by the second week after birth. Their postnatal expression profile parallels the period of intense synapse formation that occurs between days 6 and 15 (Harris et al., 1992; Fiala et al., 1998). The four SynCAM proteins continue to be expressed into adulthood, albeit at lower levels (Fig. 3b) (data not shown). At P15, additional N-glycosylated SynCAM 1 species of apparent $70-90 \mathrm{kDa}$ molecular weight are prominent and appear together with its $100 \mathrm{kDa}$ species present throughout development and into adulthood. SynCAM 3 also undergoes a developmentally regulated molecular weight shift at P15, dropping in its apparent molecular weight from 58 to $49 \mathrm{kDa}$. These results demonstrate that SynCAM proteins are coexpressed in the developing brain and therefore have the potential to share functions with SynCAM 1 during synaptogenesis and can be modified with carbohydrates in a developmentally regulated manner.

SynCAMs are synaptic membrane proteins SynCAM proteins are present at presynaptic and postsynaptic excitatory sites as detected by a pleioSynCAM antibody raised against SynCAM 1 that also recognizes conserved sequences in SynCAMs 2 and 3 (Biederer et al., 2002) (supplemental Fig. 1 , available at www.jneurosci.org as supplemental material). To investigate the distribution of individual SynCAM proteins in neurons during the peak period of synaptogenesis, we performed subcellular fractionations of rat forebrain at P9 (Fig. $4 a$ ). Each SynCAM protein was strongly enriched in synaptic plasma membranes (Fig. 4a, lane 7). The adhesion molecule $\mathrm{N}$-cadherin, which is localized to developing and mature synaptic sites (Uchida et al., 1996; Elste and Benson, 2006), served as a fractionation control. The fact that SynCAM proteins appeared enriched more strongly in purified synaptic plasma membranes than $\mathrm{N}$-cadherin indicates that they are prominently present in this fraction. Synaptophysin and Rab GDI were markers for synaptic vesicles and soluble proteins, respectively. The presence of SynCAM proteins in the crude synaptic vesicle preparation was mostly caused by nonsynaptic vesicular membranes in this fraction (supplemental Fig. 2, available at www.jneurosci.org as supplemental material). These results indicate that all four SynCAM family members are strongly enriched in synaptic membranes during the period of synapse formation.

We complemented this biochemical analysis by studying SynCAM localization in cultured neurons. In the absence of antibodies applicable for immunostaining, we expressed epitope-tagged constructs in dissociated hippocampal neurons at 7 d.i.v., when neurons in culture can rapidly form synapses (Fletcher et al., 1994). In these constructs, one extracellular flag epitope was inserted C-terminal of the third Ig-like domain to minimally interfere with putative intracellular sorting signals. In transfected neurons, SynCAM 1-flag exhibited a mostly punctate staining 
pattern with some of the signal being diffuse (Fig. $4 b$ ), similar to other synaptic membrane proteins such as syntaxin (Garcia et al., 1995). To gain better insight into the distribution of SynCAM proteins in neurons, we performed triple immunostainings of SynCAM 1-flag with the inhibitory presynaptic marker GAD65 and the excitatory presynaptic marker PSD-95 (Fig. 4b). Quantification showed that SynCAM 1-flag was sorted to both types of synaptic specializations but was preferentially detected at PSD95-labeled excitatory sites, with which $\sim 53 \%$ of SynCAM 1-flag puncta colocalized, compared with $24 \%$ of its puncta being immunopositive for GAD65. Similarly, SynCAM 2-flag exhibited a punctate pattern in hippocampal neurons and was detected at both excitatory and inhibitory sites, with $\sim 62 \%$ of SynCAM 2-flag in colocalization with PSD-95 and 31\% with GAD65 (Fig. $4 c$ ). This analysis is consistent with our observation that both excitatory and inhibitory synaptic specializations are immunopositive for endogenous SynCAMs 1, 2, and 3, which were detected by our pleioSynCAM antibody (supplemental Fig. 3, available at www.jneurosci.org as supplemental material).

To confirm that exogenously expressed SynCAM proteins indeed are sorted to synaptic sites as defined by aligned presynaptic and postsynaptic specializations, we performed triple colocalization of SynCAM 1-flag or SynCAM 2-flag with the presynaptic marker SV2 and the postsynaptic marker PSD-95 (supplemental Fig. 4, available at www.jneurosci.org as supplemental material). Approximately $45 \%$ of detected SynCAM 1-flag and 67\% of SynCAM 2-flag puncta colocalized with both markers, confirming their presence at aligned synapses (supplemental Fig. 4, available at www.jneurosci.org as supplemental material). These results are consistent with the primarily synaptic localization of endogenous SynCAMs 1, 2, and 3 (supplemental Fig. 5, available at www.jneurosci.org as supplemental material). Together, our results demonstrate that SynCAM proteins are synapse components present at excitatory as well as inhibitory synaptic specializations.

Homophilic cell adhesion is conveyed by SynCAMs 1, 2, and 3 The fact that SynCAMs are expressed in different regional patterns (Fig. $2 b, c$ ) suggested that they may confer distinct adhesive properties to neuronal populations differentially expressing SynCAM proteins. To identify these properties, we first addressed whether all SynCAM proteins mediate homophilic cell adhesion similar to SynCAM 1. Using fluorophore-labeled beads coated with each SynCAM extracellular domain, we determined whether these domains conferred bead clustering, indicative of binding to themselves (Fig. $5 a$ ). The extracellular domains of SynCAM 1, 2, and 3 caused extensive clustering of beads, consistent with previous reports for SynCAM 1 and 3 (Biederer et al., 2002; Kakunaga et al., 2005). This demonstrates that each of these three proteins, including the previously uncharacterized SynCAM 2, can engage in a homophilic interaction. In contrast, beads coated with the SynCAM 4 extracellular domain did not adhere to each other, excluding strong interactions of this protein with itself under this condition. The same SynCAM 4 extracellular domain protein conferred heterophilic binding (see below) (Fig. 6a), showing that it is properly folded and capable of engaging in its correct adhesive interactions.

To confirm that SynCAM extracellular domains engage themselves in cell adhesive trans-interactions, we individually expressed full-length SynCAMs in HEK 293 cells. The expressed proteins were tagged with CFP within their cytosolic sequence for protein detection. Cells were analyzed by fluorescence microscopy for homophilic trans-interactions of SynCAM proteins at a

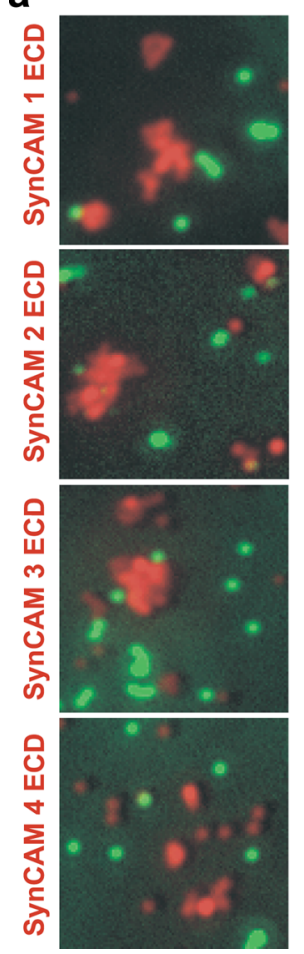

b

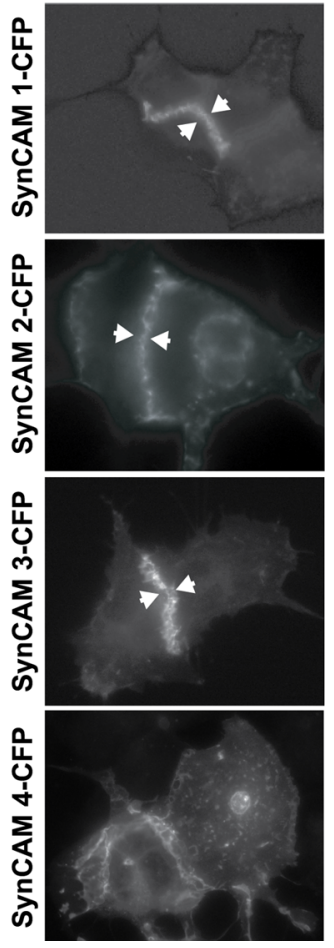

Figure 5. Homophilic cell adhesive interactions are mediated by SynCAMs 1, 2, and 3. $\boldsymbol{a}$, The extracellular domains of SynCAM 1, 2, and 3 interact homophilically. Fluorescent beads coated with the purified, heterologously expressed extracellular domains of individual SynCAM proteins as indicated on the left of the panels (red) were mixed in the presence of negative control beads coated with IgG protein (green). Bound amounts of SynCAM proteins and control lgG were comparable (data not shown). Clustering of beads shown in red displaying SynCAM 1, 2, or 3 extracellular domains demonstrates their homophilic interaction, which was not observed for SynCAM 4. Negative control beads (green) were monodisperse and not found in SynCAM bead clusters. $\boldsymbol{b}$, Homophilic SynCAM 1, 2, and 3 interactions occur in trans to mediate cell adhesion. HEK 293 cells were transfected with one of the full-length SynCAM 1, 2, 3, or 4 proteins. Proteins were tagged intracellularly with CFP, which did not interfere with plasma membrane sorting as confirmed by surface biotinylation (data not shown). Cells were analyzed by fluorescence microscopy, and localization of the indicated SynCAMs is shown in gray scale. Aggregation of SynCAM 1, 2, and 3 at sites of cell-cell contact is marked by arrowheads and demonstrates homophilic cell adhesion through trans-interactions of their extracellular domains. No homophilic cell adhesion was observed for SynCAM 4 in this assay, consistent with results in $\boldsymbol{a}$.

sites of cell-cell contact (Fig. 5b). In agreement with the bead clustering assay, we observed cell adhesive interactions of SynCAMs 1, 2, and 3. Their homophilic interaction recruited almost all detectable SynCAM proteins from the surrounding plasma membrane to sites of cell-cell contact, where they formed extended zipper-like structures. Homophilic cell adhesion was not detected for SynCAM 4, consistent with the bead clustering assay results. These results demonstrate that SynCAM 1, 2, and 3, but not 4 , are strong homophilic cell adhesion molecules and identify that SynCAMs can exhibit distinct extracellular interactions. They therefore have the potential to mediate unique functions in neuronal adhesion.

\section{Strong and specific heterophilic interactions define each SynCAM family member}

Our observations that SynCAM proteins can exert distinct extracellular interactions and are differentially expressed prompted us to examine their adhesive properties in biochemical detail. Based on our analysis of sequence conservation in the SynCAM Ig-like domains (Biederer, 2006), we hypothesized that different Syn- 
CAMs might bind each other. To address this question, we first performed affinity chromatography experiments and loaded detergent extracts from membrane preparations of rat forebrain on beads containing equal amounts of immobilized extracellular domains of each individual SynCAM family member expressed as IgG-fusion proteins (Fig. 6a). SynCAM 1 showed weak, saltsensitive homophilic binding (data not shown) (asterisks in Fig. $6 a$ indicate cross-reactive bands). In contrast, SynCAM 1 was retained strongly in a primarily salt-resistant manner on the SynCAM 2 extracellular domain (Fig. $6 a$, first row, lanes 7, 8). No binding of SynCAM 1 to other family members was detected. Reciprocally, SynCAM 2 bound strongly to the SynCAM 1 extracellular domain (Fig. $6 a$, second row, lanes 3,4 ) but not to any other SynCAM protein. SynCAM 3 engaged in a strong interaction with SynCAM 4 (Fig. 6a, third row, lanes 16, 17) in agreement with a recent study (Maurel et al., 2007; Spiegel et al., 2007). The complementary binding of brain SynCAM 4 to immobilized SynCAM 3 was not observed in this assay, possibly because of an excess of SynCAM 3 over SynCAM 4 in the brain homogenate used as starting material for binding (data not shown), which could lead to sequestration of free SynCAM 4 in the detergent extract. SynCAM 3 additionally showed weak binding to SynCAM 1 (Fig. $6 a$, third row, lanes 3, 4). The observed heterophilic SynCAM $1 / 2$ and $3 / 4$ interactions were primarily resistant to a high $800 \mathrm{~mm}$ salt washing step, indicative of their high strength and specificity. We could not detect the homophilic interactions of SynCAM 2 or 3 in this assay, possibly because affinity chromatography is a rather stringent assay of adhesive properties (Boucard et al., 2005), or because of the need for a specific interaction geometry that may be impaired in this experimental approach. Importantly, our results underline that the prominent binding of SynCAM 2 to SynCAM 1 detected by affinity chromatography appears stronger than the homophilic interaction of either of them alone. Similarly, retention of SynCAM 3 on SynCAM 4 is readily detected in contrast to SynCAM 3 homophilic binding, indicating that SynCAM 3 also prefers heterophilic interactions.

Next, we analyzed SynCAM 1 functions as a heterophilic cell adhesion molecule using full-length constructs tagged with different intracellular epitopes (Fig. 6b-d). HEK 293 cells expressing flag-tagged SynCAM proteins were mixed with cells expressing different CFP-tagged SynCAM family members. Contact sites between cells expressing different SynCAM proteins were analyzed by fluorescence microscopy. SynCAM 1 engaged SynCAM 2 in a heterophilic cell adhesive interaction (Fig. 6b), consistent with our affinity chromatographic analysis. The intercellular interaction of SynCAM 1 and 2 caused zipper-like structures to form at cell contact sites, in agreement with strong heterophilic adhesion of these two proteins. The weak binding of SynCAM 3 to SynCAM 1 detected biochemically (Fig. 6a) did not mediate cell adhesion under these conditions (Fig. $6 c$ ). Additionally, the heterophilic adhesion of SynCAM 3 and 4 was observed as zipper- or grid-like structures (Fig. 6d). As expected, no adhesive interaction of SynCAM 1 with 4 was detected (data not shown). SynCAM 1/2 and SynCAM 3/4 therefore constitute specific, cognate pairs of heterophilic neuronal adhesion molecules. Together, our analysis defines that SynCAM proteins engage each other in highly specific interactions that distinguish them individually, reminiscent of an adhesive code (Fig. 6e).

\section{SynCAM adhesion is mediated by the first two Ig-like domains and is regulated by glycosylation}

To better characterize SynCAM adhesion complexes, we aimed to identify the domains that mediate the heterophilic interactions of
SynCAM 1 (Fig. 7a). We immobilized IgG-fusion proteins corresponding to the three individual or combined Ig-like domains of SynCAM 1 and incubated them with detergent extracts from membrane preparations of rat forebrain. The extracellular partner SynCAM 2 was retained by the combined first and second Ig-like domains of SynCAM 1 as efficiently as by the full SynCAM 1 extracellular domain (Fig. $7 a$, lanes 8 vs 4 ). The combined second and third Ig-like domains of SynCAM 1, however, did not bind SynCAM 2 (lane 13). Individually, only the first Ig-like domain of SynCAM 1 exerted weak interactions with SynCAM 2 (lane 17), whereas the second and third Ig-like domains alone showed no retention, identical to negative control beads (data not shown). These results indicate that the first two Ig-like domains of SynCAMs together mediate efficient trans-interactions as depicted in Figure 6e. Notably, one individual Ig domain is $\sim 4.0 \mathrm{~nm}$ long (Davies et al., 1975). Assuming that two SynCAM extracellular domains engage each other in an extended conformation, the overall length of their complex would be approximately equivalent to four Ig-like domains in tandem flanked by the sequences at the stalk of their extracellular domains. For human SynCAM 1 and 2, these flanking sequences are 31-70 and $18-58$ aa long, respectively, depending on alternative splicing of this region (Biederer, 2006). Assuming an $\alpha$-helical conformation of these stalks, the extracellular portion of a fully extended SynCAM 1/2 complex can be predicted to have a length of $23-35 \mathrm{~nm}$.

The first two Ig-like domains of SynCAM 1 contain four predicted N-glycosylation sites (Biederer, 2006). Considering that SynCAM 1 undergoes developmentally regulated $\mathrm{N}$-glycosylation, we asked whether this modification of its Ig-like domains may affect its extracellular interactions. We performed affinity chromatographies of solubilized rat membrane proteins on the natively N-deglycosylated SynCAM 1 extracellular domain or on the control-treated extracellular domain (Fig. 7b). Enzymatic removal of N-linked carbohydrates on SynCAM 1 reduced its strong retention of SynCAM 2 and its weaker binding to SynCAM 3 each approximately threefold to fourfold. The extensive modification of SynCAM 1 with $\mathrm{N}$-linked carbohydrates therefore promotes its extracellular interactions, possibly by providing for a structural organization of the extracellular domains that fits the conformation they adopt during adhesion. Developmental changes in SynCAM 1 glycosylation therefore may serve to regulate its adhesive interactions.

\section{SynCAM 1 and 2 assemble into an adhesive synaptic complex in vivo}

Our localization and binding studies performed in vitro indicated that these heterophilic SynCAM interactions could occur at synaptic membranes in vivo. The strong heterophilic binding observed between SynCAM 1 and 2 was of particular interest because SynCAM 2 may mediate synaptic effects of SynCAM 1 (Biederer et al., 2002; Sara et al., 2005). To test whether these two adhesion molecules interact at synapses, we performed coimmunoprecipitation experiments (Fig. 8). Synaptosomes were prepared from rat brains at P15, when all SynCAM proteins are prominently expressed. Synaptosomal membrane proteins were solubilized and subjected to immunoprecipitation with a specific monoclonal antibody against the SynCAM 1 extracellular sequence. Immunoprecipitates were enriched for SynCAM 1 (Fig. 8, top, lane 3), whereas control antibodies did not precipitate SynCAM 1 or any other protein analyzed (lane 5). Importantly, SynCAM 1 antibodies coimmunoprecipitated SynCAM 2 from synaptosomal membranes (Fig. 8, second panel, lane 3) but none 


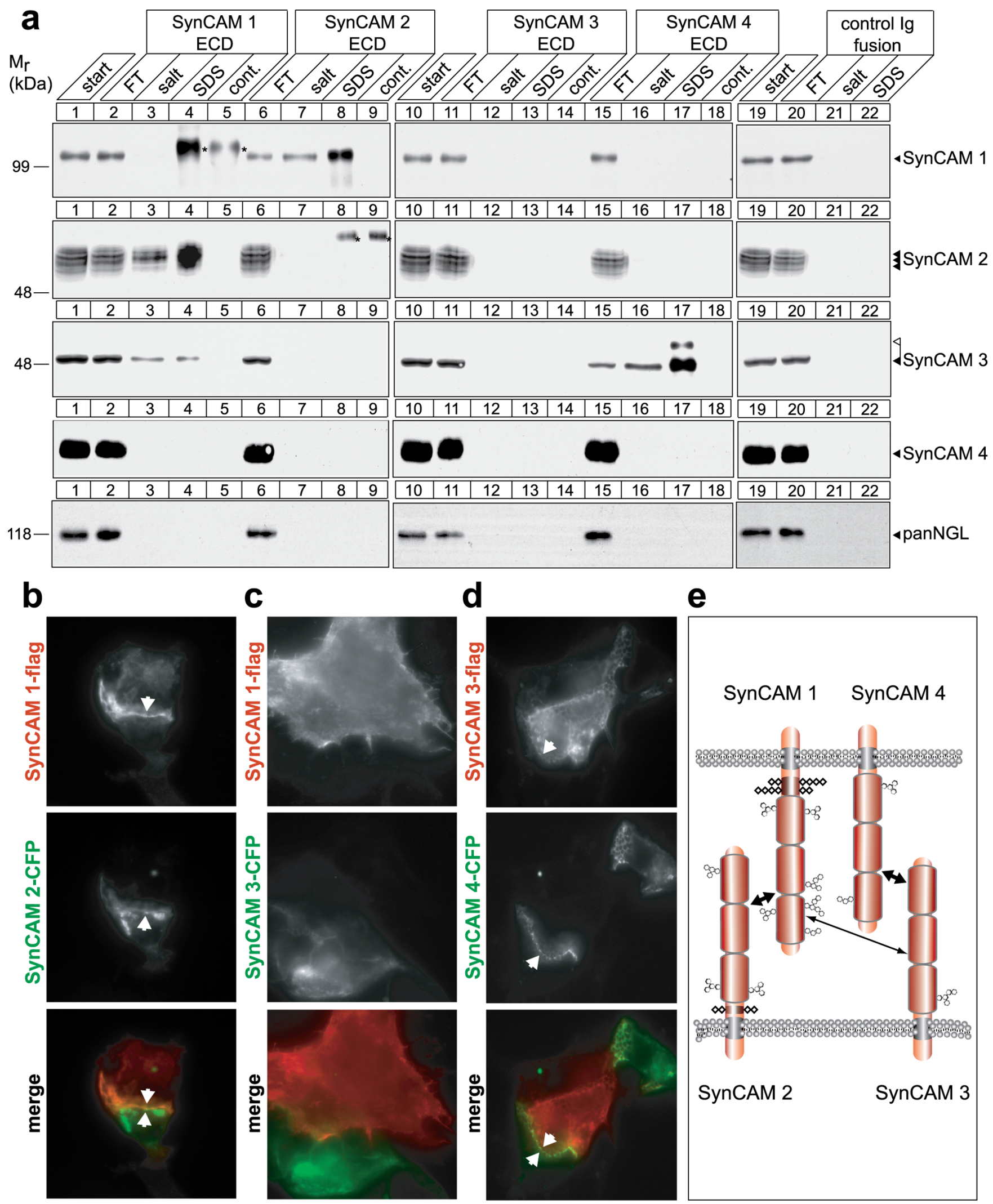

Figure 6. Specific heterophilic interactions define cognate SynCAMs to mediate cell adhesion. $\boldsymbol{a}$, Analysis of SynCAM interactions by affinity chromatography. Membrane proteins from adult rat forebrains were solubilized to obtain a detergent extract (lanes 1,10, 19) and loaded on beads containing covalently immobilized extracellular domains of SynCAM 1 (lanes 2 -4), SynCAM 2 (lanes 6-8), SynCAM 3 (11-13), SynCAM 4 (15-17), or a control lg protein (lanes 20 -22). The flow-through (FT) of each affinity chromatography was obtained, beads were washed, subsequently eluted with buffer containing high salt at $800 \mathrm{~mm}$, and finally eluted with SDS. Samples were analyzed by immunoblotting for each of the four SynCAM proteins as shown, using monoclonal chicken antibodies to detect SynCAM 1, and with an antibody detecting the NGL family of synaptic membrane proteins as negative control. To control for cross-reactivity of anti-SynCAM antibodies with SynCAM extracellular domains used as affinity matrix that were washed off the beads and were present in the eluate samples, beads containing only the purified extracellular domains were eluted with SDS, and an eluate amount equal to the respective affinity chromatography fraction was loaded (lanes 5, 9, 14, 18). Antibody cross-reactive bands are marked by asterisks. Strong heterophilic 
of the other SynCAMs or control proteins. To define whether these interactions are altered during brain development, we performed these coimmunoprecipitation experiments further in adult rat brain and determined that SynCAM 1 and 2 formed a physical complex in adult brain to an apparently identical extent as at P15 (data not shown). Notably, also in adult brain, no interactions of SynCAM 1 and 3 were observed. This coimmunoprecipitation of SynCAM 2 with SynCAM 1 is consistent with our affinity chromatography results and extends our analysis of SynCAM complex formation to native conditions in brain. The heterophilic interaction of SynCAM 1 and 2 is therefore specific and occurs throughout development in synaptosomal membranes in vivo.

\section{SynCAM 1 and 2 recruit presynaptic markers}

Do SynCAM 1 and SynCAM 2 have comparable functions at synapses? To begin to address this question, we performed mixed cocultures of hippocampal neurons with HEK 293 cells expressing either of these two proteins on their surface. To visualize transfected HEK 293 cells, we used the CFP-tagged SynCAM 1 and 2 constructs that had been used for the cell adhesion assays shown in Figure $5 b$. The activity of SynCAMs to organize presynaptic specializations was quantitatively assessed by determining the surface area of HEK 293 cells that was immunopositive for recruited presynaptic vesicle protein synapsin in coculture with neurons (Fig. 9a). SynCAM 1 expression in cocultured HEK 293 cells caused a significant increase of synapsin-positive puncta atop the cell surface compared with negative control HEK 293 cells expressing soluble CFP alone (Fig. $9 b$ ) as described previously (Biederer et al., 2002). Importantly, expression of SynCAM 2 similarly resulted in the significant detection of synapsin-

\section{$\leftarrow$}

and reciprocal binding of SynCAM 1 (first row, lanes 7, 8) and SynCAM 2 (second row, lanes 3, 4) is observed, causing their significant reduction in the respective FT fractions consistent with their high yield in the eluates. SynCAM 1 homophilic retention on its extracellular domain was weak and only detected after long immunoblot exposures (data not shown). SynCAM 3 is retained strongly in both its lower and higher molecular weight forms on SynCAM 4 (third row of panels, lanes 16, 17), and a weaker interaction of SynCAM 3 with SynCAM 1 is also observed (third row, lanes 3,4). The observed interactions were primarily salt resistant. For a discussion of lack of reciprocal SynCAM 4/3 binding, see the Results. Numbers on the left indicate positions of molecular weight markers. Arrowheads show the running positions of the indicated SynCAM proteins in their predominantly adult (black) and postnatal (white) glycosylation forms. Input and FT lanes contain $10 \%$ of the extract used for each affinity chromatography. $\boldsymbol{b}-\boldsymbol{d}$, SynCAM proteins $1 / 2$ and $3 / 4$ interact in trans to confer heterophilic cell adhesion. HEK 293 cells were individually transfected with constructs encoding the indicated epitope-tagged SynCAM proteins, and cells were mixed in a 1:1 ratio. Localization of individual SynCAMs at sites of cell-cell contact was analyzed by fluorescence microscopy and is shown in gray scale as indicated. Bottom, Merged images (flag-tagged constructs, red; (FP-tagged constructs, green). To assess interactions of SynCAM 1, HEK 293 cells were transfected with SynCAM 1-flag and mixed with HEK 293 cells expressing either SynCAM 2-CFP (b) or SynCAM 3-CFP (c). b , SynCAM 1/2 heterophilic cell-cell interactions were readily detected, consistent with their strong biochemical interaction observed in $\boldsymbol{a}$. c, No strong cell adhesive interactions were demonstrated in this assay for SynCAM 1 and 3. $\boldsymbol{d}$, To assess interactions of SynCAM 4, HEK 293 cells transfected with SynCAM 4-CFP were mixed with HEK 293 cells expressing SynCAM 3-flag, and SynCAM 3/4 heterophilic cell-cell interactions were detected. Arrowheads mark SynCAM protein aggregation at sites of cell-cell contact. All constructs were epitope tagged within their intracellular sequences, which did not interfere with plasma membrane sorting as described in Figure $5 b$. The SynCAM 1-flag/1-CFP homophilic interaction served as positive control (data not shown).e, Model of heterophilic interactions between SynCAM family members. SynCAM 1 and 2 as well as 3 and 4 form two strong cognate cell adhesion pairs. In addition, SynCAM 1 and 3 can bind more weakly. The additional homophilic interactions of SynCAM 1, 2, and 3 are not shown. Ig-like domains are represented as barrels, N-linked carbohydrates as hexagons, and 0 -linked carbohydrates as rhombi. Predicted glycosylation sites in SynCAM proteins are drawn to scale (Biederer, 2006).
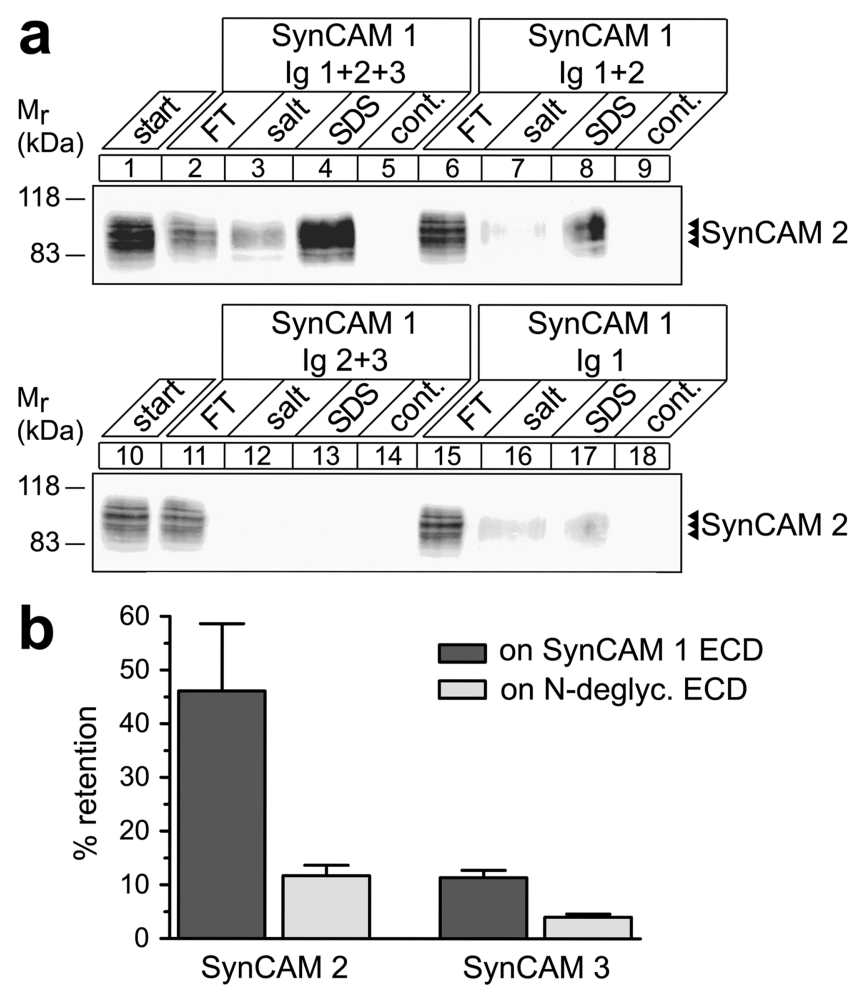

Figure 7. Heterophilic SynCAM binding is mediated by the first two Ig-like domains and is controlled by N-glycosylation. $\boldsymbol{a}$, Mapping of SynCAM Ig-like domain interactions by affinity chromatography. Membrane proteins from adult rat forebrains were solubilized (lanes 1, 10) and loaded on beads containing equal amounts of covalently immobilized fusion proteins corresponding to the indicated SynCAM $1 \mathrm{lg}$-like domains. The flow-through (FT) of each affinity chromatography was obtained, beads were washed, subsequently eluted with buffer containing high salt at $800 \mathrm{~mm}$, and finally eluted with SDS. Eluates from beads containing only the immobilized fusion proteins served as negative controls for antibody cross-reactivity. Samples were analyzed by immunoblotting for SynCAM 2, which bound as strongly to the first three and first two Ig-like domains of SynCAM 1 (lanes 8 vs 4), but only weakly to its first lg-like domain (lane 17), and not to either of the other Ig-like domains in combination (lane 13) or alone (data not shown). No binding of SynCAM 2 to beads containing control lgG was observed (data not shown). The numbers on the left indicate positions of molecular weight markers. Input and FT lanes contain $10 \%$ of the extract amount used for each affinity chromatography. $\boldsymbol{b}$, Interactions of SynCAM 1 with SynCAM 2 are controlled by N-glycosylation of SynCAM $1 \mathrm{lg}$-like domains. Membrane proteins from adult rat forebrains were solubilized and loaded on beads containing the full-length SynCAM 1 extracellular domain treated under native conditions either without or with PNGase F to remove N-linked carbohydrates. Binding was analyzed by quantitated immunoblotting. Control SynCAM 1 efficiently retained SynCAM 2, but natively N-deglycosylated SynCAM 1 exerted fourfold reduced binding. Consistent with a weaker interaction of SynCAM 3, it was retained approximately fourfold less on the control SynCAM 1 extracellular domain, and this retention was also reduced by deglycosylation of SynCAM 1. Error bars represent SDs $(n=3)$.

positive puncta atop HEK 293 cells. These results indicate that both SynCAM 1 and 2 organize presynaptic specializations in this mixed coculture assay. We also analyzed the extracellularly flagtagged SynCAM 1 and 2 proteins in this assay and observed that their activity was indistinguishable from the described SynCAM constructs with native extracellular sequences (data not shown). This demonstrates that they are functional and suitable for the localization studies shown in Figure $4, b$ and $c$, and below.

\section{SynCAM 1 and 2 recruit each other into adhesive neuronal complexes}

Do the heterophilic interactions of SynCAM 1 and 2 only occur at stable synapses or can they recruit SynCAMs to sites of contacts with differentiating neurons? We analyzed this question in a modified coculture system of dissociated hippocampal neurons 
expressing extracellularly flag-tagged SynCAM 2 with HEK 293 cells expressing CFP-tagged SynCAM 1 (Fig. 9c). After $2 \mathrm{~d}$ of coculture, neuronal SynCAM 2-flag was strongly recruited to sites of contact with SynCAM 1 expressed in HEK 293 cells (Fig. $9 c)$. Serial reconstruction of confocal images demonstrated that the SynCAM 2-expressing neurons formed extensive membrane contacts containing concentrated amounts of neuronal SynCAM 2-flag that enveloped SynCAM 1 expressing HEK 293 cells (Fig. $9 c$, second and third rows). Immunostaining for the presynaptic marker synapsin atop the HEK 293 cells expressing SynCAM 1 served as positive control for its activity to organize presynaptic specializations in these coculture experiments (Fig. $9 c$, fourth row). The staining of HEK 293-expressed SynCAM 1 does not extensively mirror the staining of presynaptic SynCAM 2-flag in the contacting neuron, showing areas lacking overlap with neuronal SynCAM 2-flag. This can be expected because of the assay design, with multiple neurons in addition to the one transfected neuron contacting a given HEK cell that all can contribute neuronal interaction partners with HEK cell-expressed SynCAM 1. Together, these results reveal that the adhesive SynCAM code is instructive for the specific assembly of the SynCAM 1/2 complex across membranes.

To better understand the dynamics of synaptic SynCAM assembly, we next tested whether SynCAM 1 and 2 can also operate in reverse. Following the same paradigm as outlined above, we analyzed hippocampal neurons expressing SynCAM 1-flag that were cocultured with HEK 293 cells expressing CFP-tagged SynCAM 2 for SynCAM recruitment (Fig. 9d). SynCAM 2 presented from the HEK 293 cell surface was able to assemble into a complex with neuronal SynCAM 1-flag. In conjunction, HEK cellexpressed SynCAM 2 mediated synapsin recruitment from the surrounding neurons. Specificity of SynCAM 1/2 assembly was confirmed by the inability of negative control HEK 293 cells expressing CFP alone to assemble neuronal SynCAM 1 complexes after surface contact, in parallel to the lack of synapsin clusters atop these control HEK 293 cells (Fig. 9e). Together, we demonstrate that the interactions of SynCAM 1 and 2 on neuronal surfaces are reciprocal and recruit each other into adhesive complexes.

\section{SynCAM 1 and 2 promote functional synapses}

Based on our analysis of SynCAMs in cocultures, we aimed to understand whether SynCAM 1 and 2 also operate between neurons to organize synapses and control synaptic function. To address these questions, we overexpressed SynCAM 1 or 2 in dissociated hippocampal neurons. First, we determined whether SynCAM 1 and 2 alter the density of functional presynaptic terminals formed between neurons in culture (Fig. 10a,b). Hippocampal neurons were transfected at the beginning of the peak period of synaptogenesis in culture at 7 d.i.v. with vectors encoding wild-type SynCAM proteins and soluble GFP. We used a low-efficiency transfection method to increase SynCAM expression only in a small subset of neurons and, hence, to elevate SynCAM protein amounts only in one of the cells that is in contact with other neurons. Four days later, at the height of endogenous synaptogenesis, we briefly depolarized the neurons and labeled all active presynaptic specializations by uptake of antibodies directed against the luminal domain of synaptotagmin 1 into recycling synaptic vesicles (Matteoli et al., 1992; Biederer and Scheiffele, 2007). Analysis of the density of puncta positive for synaptotagmin 1 uptake was performed along the dendrites of SynCAM-overexpressing pyramidal neurons identified through their GFP signal (Fig. 10a). This allowed us to directly determine

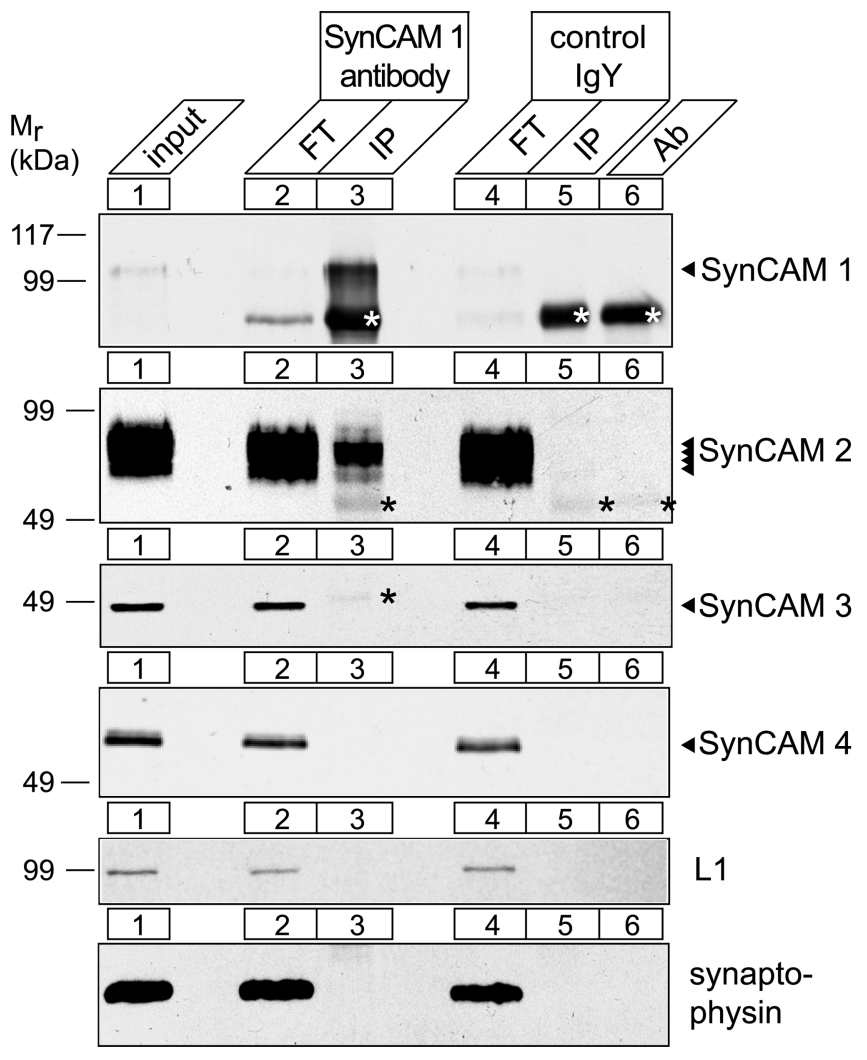

Figure 8. SynCAM 1 and 2 form a specific adhesive complex in synaptic membranes in vivo. Synaptosomes were prepared from rat forebrain at P15 during the peak of SynCAM expression and synaptogenesis. Synaptosomal membrane proteins were extracted with detergent (lane 1) and immunoprecipitated with specific antibodies against the extracellular domain of SynCAM 1 (lanes 2,3) or control antibodies (lanes 4, 5). The flow-through (FT) of each immunoprecipitation was obtained, and immunoprecipitates (IP) were collected on beads, washed, and eluted with SDS. The same amount of antibody used for the immunoprecipitation was loaded to identify cross-reactive bands recognized by secondary antibodies (lane 6), which are marked by asterisks. Fractions were probed for each of the four SynCAM proteins as indicated, and with an antibody detecting the L1 Ig superfamily member and the synaptic vesicle protein synaptophy$\sin$ as negative control membrane proteins. SynCAM 1 coimmunoprecipitated SynCAM 2 from synaptosomes but no other SynCAM family member (lane 3). Numbers on the left indicate positions of molecular weight markers. Input and FT lanes contain $4 \%$ of the extract amount used for each immunoprecipitation.

the number of active presynaptic terminals formed atop neurons with elevated SynCAM expression (Fig. 10b). Neurons expressing only GFP served as negative control. Postsynaptic SynCAM 2 overexpression resulted in a significant increase by $34 \pm 9 \%$ in the density of presynaptic terminals containing recycling synaptic vesicles along neuronal dendrites. SynCAM 1 overexpression caused a trend to increased presynaptic terminal density, which was not statistically significant. Our results show that postsynaptic SynCAM 1 and 2 engage incoming axons in transsynaptic interactions, which in turn causes contacting presynaptic neurons to display more terminals.

To determine whether these new presynaptic terminals organized by SynCAMs are functional, we transduced hippocampal neurons at 6-8 d.i.v. with expression vectors encoding fulllength SynCAM 1 or 2 proteins and soluble GFP. Miniature EPSCs (mEPSC) were recorded from GFP-expressing neurons 12-24 h later (Fig. 10c,d). SynCAM 1 overexpression increased mEPSC frequency when compared with untransfected control neurons in the same culture (Fig. 10d) or to GFP-transfected neurons (data not shown) as reported previously (Biederer et al., 
2002; Sara et al., 2005). Importantly, overexpression of SynCAM 2 in neurons similarly enhanced mEPSC frequency approximately threefold (Fig. 10d). mEPSCs amplitudes were not significantly changed (supplemental Fig. 6, available at www.jneurosci.

org as supplemental material). This indicates a substantial increase in excitatory synapse number and function caused by SynCAM overexpression.

Together, our results demonstrate that SynCAM proteins bind each other in specific heterophilic patterns and identify SynCAM 1 and 2 as components of cognate transsynaptic complexes. Concurringly, the components of the SynCAM 1/2 complex each organize functional synapses and potentiate excitatory synaptic transmission.

\section{Discussion}

Our results reveal SynCAMs as synaptic membrane proteins that assemble into specific adhesion complexes, with the cognate partners SynCAM 1 and 2 recruiting each other to neuronal membrane sites. Notably, both SynCAM 1 and 2 can organize synapses and promote excitatory synaptic transmission. Together, we demonstrate that molecularly defined SynCAM adhesion complexes exist, and that SynCAM proteins participate in synaptic organization and contribute to synaptic function.

The properties of SynCAMs described here are consistent with their transsynaptic localization and point to a common synaptic function. In the hippocampus, all SynCAMs are expressed mostly by neurons during the peak period of synaptogenesis around the second postnatal week. SynCAM 1 and 2 are present at synapses, and the trans-interaction of their first two Iglike domains suggests an extended SynCAM complex that would fit the $20 \mathrm{~nm}$ cleft width of central synapses (Schikorski and Stevens, 1997; Ahmari and Smith, 2002). SynCAMs are ubiquitously expressed throughout the brain, indicating a wide-ranging function, but also display notable differences in their regional profiles, consistent with nonoverlapping roles in distinct neuronal populations. Additionally, they show the gradual developmental increase in brain expression typical for neuroligins and other synaptic proteins (Song et al., 1999). SynCAM proteins remain expressed throughout adulthood (A.I.F., M.R.A., and T.B., unpublished results), when they may function in synapse maturation or stabilization.

An intriguing biochemical property of SynCAM proteins is their highly distinct heterophilic adhesion pattern. In drawing
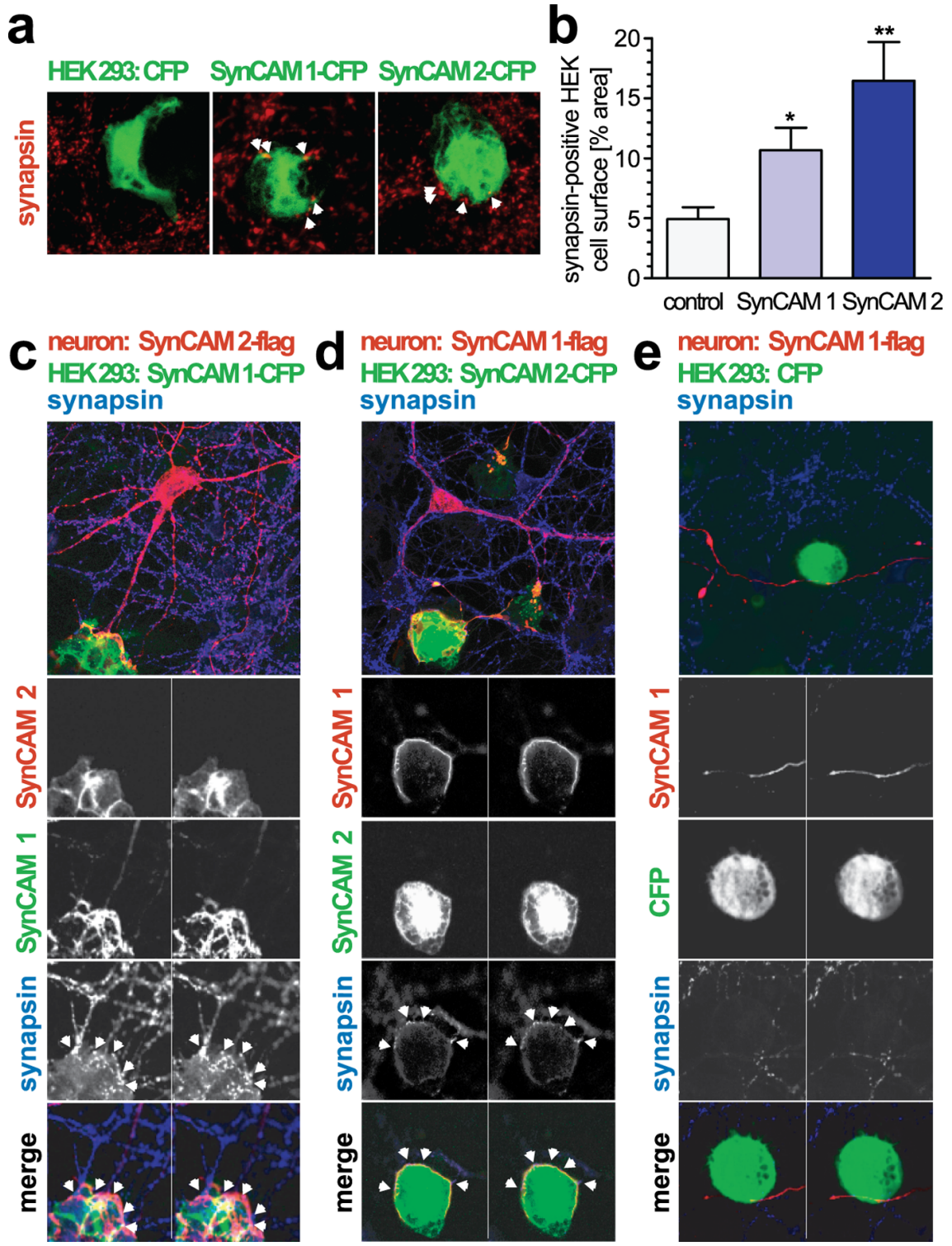

Figure 9. SynCAM 1 and 2 recruit presynaptic marker proteins and engage each other in adhesive neuronal complexes. $\boldsymbol{a}$, SynCAM 1 and 2 recruit synapsin in a mixed coculture assay. HEK 293 cells expressing CFP-tagged SynCAM 1 or CFP-tagged SynCAM 2 were seeded atop dissociated hippocampal cultures at 9 d.i.v. HEK 293 cells expressing soluble CFP alone served as negative control. Cocultures were analyzed at 11 d.i.v. by confocal microscopy for localization of the presynaptic vesicle marker synapsin (red) and CFP (green). Merged stacks of serial optical sections are shown. Synapsin puncta were detected atop SynCAM 1 - and SynCAM 2-expressing HEK 293 cells and are marked with white arrowheads in the images shown. $\boldsymbol{b}$, Expression of SynCAM 1 or SynCAM 2 in HEK 293 cells cocultured with hippocampal neurons caused a significant increase of synapsin-positive puncta covering the cell surface area compared with control HEK 293 cells expressing CFP alone. The activity of both SynCAMs was similar (CFP alone, $4.9 \pm 1.0 \%$ synapsin area coverage, $n=15$ cells; SynCAM 1-CFP, $10.7 \pm 1.9 \%, p=0.011$ relative to CFP, $n=15$ cells; SynCAM 2-CFP, $16.5 \pm 3.2 \%, p=0.005$ relative to (FP, $n=20$ cells). Statistical analyses were performed using unpaired $t$ tests with two-tailed $p$ values. $c$, SynCAM 1 recruits and retains neuronal SynCAM 2 in adhesive neuronal complexes. Dissociated hippocampal neurons were transfected at 7 d.i.v. with flag-tagged SynCAM 2 to allow its visualization by immunostaining. HEK 293 cells expressing CFP-tagged SynCAM 1 were seeded atop these hippocampal cultures at 9 d.i.v., and cocultures were analyzed at 11 d.i.v. by confocal microscopy for localization of both SynCAM proteins and the presynaptic vesicle marker synapsin. Optical sections of each $1 \mu \mathrm{m}$ thickness were obtained. The top panel shows, for a single optical section, the merged image (SynCAM 2-flag, red; SynCAM 1-CFP, green; synapsin, blue). The four panels at the bottom show for two serial optical sections the indicated individual immunostainings in grayscale and the triple merged image in color. White arrowheads mark synapsin puncta formed atop the SynCAM 1-expressing HEK 293 cell. $\boldsymbol{d}$, SynCAM 2 reciprocally recruits neuronal SynCAM 1 into adhesive complexes. Analysis was performed as described in cin cocultures of hippocampal neurons expressing flag-tagged SynCAM 1 and HEK 293 cells expressing CFP-tagged SynCAM 2. The top panel shows, for a single optical section, the merged staining of the three indicated proteins (SynCAM 1-flag, red; SynCAM 2-CFP, green; synapsin, blue). The panels below show two serial optical sections obtained after immunostaining for the indicated proteins as described in c. $\boldsymbol{e}$, Control HEK 293 cells do not retain SynCAM proteins. Analysis was performed as described in c in cocultures of hippocampal neurons expressing flag-tagged SynCAM 1 and HEK 293 cells expressing CFP alone. After surface contact of neurons expressing SynCAM 1, no synapsin retention or SynCAM complex formation was observed atop HEK 293 cells. 
the first comprehensive map of SynCAM interactions, we observed strong heterophilic interactions of SynCAM 1/2 and SynCAM 3/4, as well as homophilic adhesion for SynCAM 1, 2, and 3 but not SynCAM 4. SynCAM 3, also termed CADM3 and Necl-1, had been reported previously to bind SynCAM 1 after heterologous expression (Shingai et al., 2003; Kakunaga et al., 2005). Our biochemical analysis confirms this interaction, which however does not appear to convey strong binding in vitro or to occur to a notable extent in vivo. The specific and strong heterophilic interactions between SynCAM family members $1 / 2$ and 3/4 therefore likely define each SynCAM functionally in neurons and are a striking property reminiscent of a neuronal adhesive code. Furthermore, SynCAM 1 and 2 drive the recruitment of each other to sites of neuronal contact, demonstrating that their transsynaptic interaction can occur in differentiating neurons. The heterophilic binding of SynCAM 3 and 4 shown here was also reported recently in a study of myelination in the peripheral nervous system (Maurel et al., 2007; Spiegel et al., 2007). This heterophilic specificity distinguishes SynCAMs from the neurexin-neuroligin system, which is mainly controlled by alternative splicing along with certain isoform preferences (Ichtchenko et al., 1996; Boucard et al., 2005; Chih et al., 2006; Comoletti et al., 2006; Graf et al., 2006). SynCAM sequences and domain organization are highly conserved within this protein family (Biederer, 2006), and the molecular basis for the specificity of SynCAM binding is not yet understood because structural information is lacking. However, it is of interest that SynCAMs are expressed as complex glycoproteins, with SynCAM 1 and 3 undergoing a developmentally regulated glycosylation that is unique among synaptic adhesion molecules. The fact that N-glycosylation of the SynCAM 1 Ig-like domains promotes its binding to SynCAM 2 points to a role of this modification in regulating the strength and specificity of SynCAM adhesion. The mechanisms providing for such differential SynCAM glycosylation in a developmentally regulated manner are presently unknown.

Both SynCAM 1 and 2 are highly expressed in the hippocampus (M.R.A. and T.B., unpublished results), indicating their functional relevance in this region. This motivated us to determine their roles in hippocampal neurons and synapses. In addition, the analysis of synaptic functions of SynCAM 1 in hippocampal neurons (Biederer et al., 2002; Sara et al., 2005) provided a framework for our studies. We investigated synaptic effects by overexpressing individual SynCAM proteins to overcome the problems that loss-of-function approaches pose because of redundancy between parallel synaptic adhesions systems. Three results now identify that the heterophilic adhesion molecules SynCAM 1 and SynCAM 2 can organize synapses and contribute to their function. First, both SynCAM 1 and SynCAM 2 expressed in HEK 293 cells recruit presynaptic marker proteins to contact sites with hippocampal neurons. Second, elevated SynCAM 2 in dendritic membranes increases the density of functional presynaptic terminals atop these dendrites as observed by visualizing recycling synaptic vesicles. Third, overexpression of either SynCAM 1 or SynCAM 2 in neurons increases mEPSC frequency, consistent with SynCAMs promoting functional excitatory synaptic transmission. It remains presently unclear how SynCAM 1 promotes mEPSCs when exogenously expressed in hippocampal neurons without significantly increasing the number of presynaptic terminals between neurons. This could be caused by effects on the presynaptic release machinery in contacting neurons, or because of a conceivable conversion of existing silent to functional synapses by postsynaptic SynCAM 1. The fact that SynCAM 1 expression in postsynaptic neurons only provides
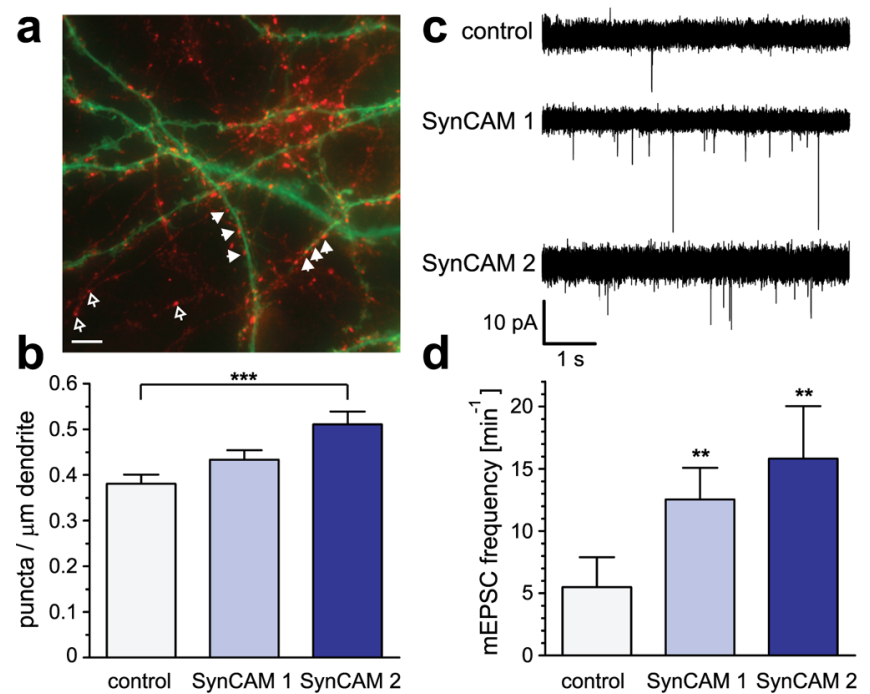

Figure 10. Components of the SynCAM 1/2 complex promote synapse differentiation and synaptic transmission between hippocampal neurons. $\boldsymbol{a}$, Analysis of postsynaptic SynCAM effects on the formation of presynaptic terminals. Dissociated hippocampal neurons were transfected at 7 d.i.v. with expression vectors for soluble GFP (green) alone or in combination with SynCAM proteins. Four days after transfection, neurons were briefly depolarized, and recycling synaptic vesicles were labeled by uptake of antibodies directed against the luminal domain of synaptotagmin 1 (red). The image shows a neuron coexpressing soluble GFP with SynCAM 1. Filled arrowheads indicate presynaptic terminals containing recycling synaptic vesicles labeled after synaptotagmin 1 uptake (red) found atop dendrites of a transfected neuron (green). Open arrowheads point to presynaptic terminals formed between neighboring untransfected neurons. The cell body of the transfected neuron is to the right and not shown. Scale bar, $5 \mu \mathrm{m} . \boldsymbol{b}$, Postsynaptic overexpression of SynCAM 2 promotes formation of presynaptic terminals. The density of synaptotagmin 1 antibody-labeled puncta on proximal dendrites of transfected pyramidal neurons was analyzed after brief depolarization as described in $\boldsymbol{a}$. Compared with control neurons, postsynaptic SynCAM 1 overexpression caused a $14 \pm 7 \%$ increase in presynaptic terminal density that was not statistically significant ( $p=0.074)$. SynCAM 2 overexpression resulted in a significant increase of presynaptic terminal density by $34 \pm 9 \%$ compared with control neurons ( $p=0.0002$ ). For image analysis, an average dendrite length of $290 \mu \mathrm{m}$ was selected per transfected pyramidal neuron starting from the first branch point of proximal dendrites. Results were obtained in independent experiments with neurons expressing only GFP as control (total 13 neurons; $3990 \mu$ m dendrite length), with neurons expressing both GFP and SynCAM 1 (14 neurons; $3770 \mu$ m dendrite), or with neurons expressing both GFP and SynCAM 2 (12 neurons; $3580 \mu \mathrm{m}$ dendrite). Statistical analyses were performed using unpaired $t$ tests with two-tailed $p$ values. c, Postsynaptic overexpression of SynCAM 1 and 2 promotes excitatory synaptic transmission of dissociated hippocampal neurons in culture. Five superimposed representative traces show mEPSCs of untreated control neurons and neurons overexpressing SynCAM 1 or SynCAM 2. Coexpression of GFP was used to identify transduced neurons. mEPSC events are recorded at higher frequencies from SynCAM 1 and SynCAM 2 overexpressing neurons than from untransduced control neurons. Expression was performed using the Semliki forest virus system for transduction of neurons. $\boldsymbol{d}$, SynCAM 1 and SynCAM 2 overexpression significantly increase mEPSC frequencies. Overexpression of SynCAM 1 $\left(n=14 ; f=12.5 \pm 2.5 \mathrm{~min}^{-1} ; p=0.001\right)$ and SynCAM2 $\left(n=11 ; f=15.8 \pm 4.2 \mathrm{~min}^{-1} ; p=\right.$ 0.004 ) caused a significant increase in $m E P S C$ frequencies compared with untransduced control neurons $\left(n=19 ; \mathrm{f}=5.5 \pm 2.4 \mathrm{~min}^{-1}\right)$. Expression of GFP alone did not cause a significant difference (data not shown). Statistical analysis was performed using a Mann-Whitney $U$ test with one-tailed $p$ value.

for a trend toward more presynaptic specializations, whereas it causes the significant recruitment of presynaptic markers when expressed in HEK 293 cells, is possibly a result of the high surface expression of heterologous cells and their lack of the endogenous SynCAM amounts found in neurons, providing for a more pronounced readout in the mixed coculture assays.

In comparing SynCAM activities, we noted that increasing SynCAM 2 amounts appeared more effective than elevating SynCAM 1. It is interesting to speculate that SynCAM 2 availability may set a threshold that determines physiological effects of SynCAM-mediated synaptic organization. Consistent with this 
hypothesis, SynCAM 1 appears somewhat less synaptic than SynCAM 2, indicating that it may be constitutively available on neurite membranes for possible recruitment by SynCAM 2 to organize nascent synaptic sites and potentially align them. This hypothesized role of SynCAM 1 would be analogous to nonsynaptic neuroligin 1 providing hotspots of synapse formation on dendrites (Gerrow et al., 2006). However, such analyses of SynCAM complex assembly will have to await the ultrastructural subsynaptic localization of each SynCAM family member, and determining the extent of their presynaptic versus postsynaptic localization will provide insight into the structure and function of SynCAM complexes. Although SynCAMs are found at both presynaptic and postsynaptic membranes (Biederer et al., 2002), lateral cis-interactions may occur in addition to the binding of SynCAMs in trans-interactions, which could serve to regulate their transsynaptic interactions similar as described previously for neurexins and neuroligins (Taniguchi et al., 2007) and for cadherin-mediated adhesion (Tanaka et al., 2000). With respect to transsynaptic functions of SynCAM complexes, it is notable that neurexins also are found at both presynaptic and postsynaptic membranes (Taniguchi et al., 2007). Because the protein interaction motifs in the cytosolic SynCAM and neurexin sequences are highly conserved (our unpublished observations), the SynCAM and neurexin/neuroligin adhesion systems may therefore engage similar intracellular partners on both sides of the synaptic cleft to organize synapses.

Together, we identify the four SynCAM proteins as a family of neuronal adhesion molecules defined by highly specific heterophilic interactions and characterize the SynCAM 1/2 complex as an asymmetric synaptic adhesion system regulated by $\mathrm{N}$-glycosylation. We demonstrate that SynCAM 1 and 2 proteins are localized to synapses, act across membranes to organize synaptic sites, and promote synaptic transmission. This study therefore introduces SynCAMs as components of a novel heterophilic transsynaptic system. How do SynCAM molecules cooperate with other transsynaptic interactions at the large variety of synapses found in the CNS? It is intriguing to hypothesize a balance between adhesion systems such as SynCAMs that could guide synaptic development and organization, those that affect both synapse organization and excitatory/inhibitory specificity, such as neurexins and neuroligins, as well as others that promote synapse maturation such as $\mathrm{N}$-cadherins. These transsynaptic interactions are likely rather complex considering the additional roles of postsynaptic SALM (synaptic cell adhesion-like molecule) and NGL proteins and EphB receptors in neuronal and synaptic differentiation (Kayser et al., 2006; Kim et al., 2006; Ko et al., 2006; Wang et al., 2006) and may ultimately not only differentiate but also specify maturing synapses (Benson et al., 2001). On this network level, SynCAM expression differences between neuronal populations combined with their precise heterophilic interaction patterns could contribute to the specificity of synaptic connectivity in addition to organizing individual synapses.

\section{References}

Ahmari SE, Smith SJ (2002) Knowing a nascent synapse when you see it. Neuron 34:333-336.

Akins MR, Biederer T (2006) Cell-cell interactions in synaptogenesis. Curr Opin Neurobiol 16:83-89.

Anderson TR, Shah PA, Benson DL (2004) Maturation of glutamatergic and GABAergic synapse composition in hippocampal neurons. Neuropharmacology 47:694-705.

Benson DL, Colman DR, Huntley GW (2001) Molecules, maps and synapse specificity. Nat Rev Neurosci 2:899-909.

Biederer T (2006) Bioinformatic characterization of the SynCAM family of immunoglobulin-like domain-containing adhesion molecules. Genomics $87: 139-150$.

Biederer T, Scheiffele P (2007) Mixed-culture assays for analyzing neuronal synapse formation. Nat Protoc 2:670-676.

Biederer T, Sara Y, Mozhayeva M, Atasoy D, Liu X, Kavalali ET, Südhof TC (2002) SynCAM, a synaptic adhesion molecule that drives synapse assembly. Science 297:1525-1531.

Boucard AA, Chubykin AA, Comoletti D, Taylor P, Sudhof TC (2005) A splice code for trans-synaptic cell adhesion mediated by binding of neuroligin 1 to alpha- and beta-neurexins. Neuron 48:229-236.

Chih B, Engelman H, Scheiffele P (2005) Control of excitatory and inhibitory synapse formation by neuroligins. Science 307:1324-1328.

Chih B, Gollan L, Scheiffele P (2006) Alternative splicing controls selective trans-synaptic interactions of the neuroligin-neurexin complex. Neuron 51:171-178.

Comoletti D, Flynn RE, Boucard AA, Demeler B, Schirf V, Shi J, Jennings LL, Newlin HR, Sudhof TC, Taylor P (2006) Gene selection, alternative splicing, and post-translational processing regulate neuroligin selectivity for beta-neurexins. Biochemistry 45:12816-12827.

Davies DR, Padlan EA, Segal DM (1975) Three-dimensional structure of immunoglobulins. Annu Rev Biochem 44:639-667.

Davis GW, Schuster CM, Goodman CS (1997) Genetic analysis of the mechanisms controlling target selection: target-derived Fasciclin II regulates the pattern of synapse formation. Neuron 19:561-573.

Dean C, Scholl FG, Choih J, DeMaria S, Berger J, Isacoff E, Scheiffele P (2003) Neurexin mediates the assembly of presynaptic terminals. Nat Neurosci 6:708-716.

DiCiommo DP, Bremner R (1998) Rapid, high level protein production using DNA-based Semliki Forest virus vectors. J Biol Chem 273:18060-18066.

Elste AM, Benson DL (2006) Structural basis for developmentally regulated changes in cadherin function at synapses. J Comp Neurol 495:324-335.

Fiala JC, Feinberg M, Popov V, Harris KM (1998) Synaptogenesis via dendritic filopodia in developing hippocampal area CA1. J Neurosci 18:8900-8911.

Fletcher TL, De Camilli P, Banker G (1994) Synaptogenesis in hippocampal cultures: evidence indicating that axons and dendrites become competent to form synapses at different stages of neuronal development. J Neurosci 14:6695-6706.

Fujita E, Kouroku Y, Ozeki S, Tanabe Y, Toyama Y, Maekawa M, Kojima N, Senoo H, Toshimori K, Momoi T (2006) Oligo-asthenoteratozoospermia in mice lacking RA175/TSLC1/SynCAM/IGSF4A, a cell adhesion molecule in the immunoglobulin superfamily. Mol Cell Biol 26:718-726.

Garcia EP, McPherson PS, Chilcote TJ, Takei K, De Camilli P (1995) rbSec1A and B colocalize with syntaxin 1 and SNAP- 25 throughout the axon, but are not in a stable complex with syntaxin. J Cell Biol 129:105-120.

Gerrow K, El-Husseini A (2006) Cell adhesion molecules at the synapse. Front Biosci 11:2400-2419.

Gerrow K, Romorini S, Nabi SM, Colicos MA, Sala C, El-Husseini A (2006) A preformed complex of postsynaptic proteins is involved in excitatory synapse development. Neuron 49:547-562.

Gorman C (1985) DNA cloning. A practical approach. Oxford: IRL.

GrafER, Zhang X, Jin S-X, Linhoff MW, Craig AM (2004) Neurexins induce differentiation of GABA and glutamate postsynaptic specializations via neuroligins. Cell 119:1013-1026.

Graf ER, Kang Y, Hauner AM, Craig AM (2006) Structure function and splice site analysis of the synaptogenic activity of the neurexin-1 $\beta$ LNS domain. J Neurosci 26:4256-4265.

Harlow E, Lane D (1999) Using antibodies: a laboratory manual. Cold Spring Harbor, NY: Cold Spring Harbor Laboratory.

Harris KM, Jensen FE, Tsao B (1992) Three-dimensional structure of dendritic spines and synapses in rat hippocampus (CA1) at postnatal day 15 and adult ages: implications for the maturation of synaptic physiology and long-term potentiation. J Neurosci 12:2685-2705.

Hayashi Y, Matsui H, Takagi T (1989) Membrane protein molecular weight determined by low-angle laser light-scattering photometry coupled with high-performance gel chromatography. Methods Enzymol 172:514-528.

Ichtchenko K, Hata Y, Nguyen T, Ullrich B, Missler M, Moomaw C, Südhof TC (1995) Neuroligin 1: a splice site-specific ligand for beta-neurexins. Cell 81:435-443. 
Ichtchenko K, Nguyen T, Südhof TC (1996) Structures, alternative splicing, and neurexin binding of multiple neuroligins. J Biol Chem 271:2676-2682.

Jones DH, Matus AI (1974) Isolation of synaptic plasma membrane from brain by combined flotation-sedimentation density gradient centrifugation. Biochim Biophys Acta 356:276-287.

Kakunaga S, Ikeda W, Itoh S, Deguchi-Tawarada M, Ohtsuka T, Mizoguchi A, Takai Y (2005) Nectin-like molecule-1/TSLL1/SynCAM3: a neural tissue-specific immunoglobulin-like cell-cell adhesion molecule localizing at non-junctional contact sites of presynaptic nerve terminals, axons and glia cell processes. J Cell Sci 118:1267-1277.

Kayser MS, McClelland AC, Hughes EG, Dalva MB (2006) Intracellular and trans-synaptic regulation of glutamatergic synaptogenesis by EphB receptors. J Neurosci 26:12152-12164.

Kim S, Burette A, Chung HS, Kwon SK, Woo J, Lee HW, Kim K, Kim H, Weinberg RJ, Kim E (2006) NGL family PSD-95-interacting adhesion molecules regulate excitatory synapse formation. Nat Neurosci 9:1294-1301.

Ko J, Kim S, Chung HS, Kim K, Han K, Kim H, Jun H, Kaang BK, Kim E (2006) SALM synaptic cell adhesion-like molecules regulate the differentiation of excitatory synapses. Neuron 50:233-245.

Kuramochi M, Fukuhara H, Nobukuni T, Kanbe T, Maruyama T, Ghosh HP, Pletcher M, Isomura M, Onizuka M, Kitamura T, Sekiya T, Reeves RH, Murakami Y (2001) TSLC1 is a tumor-suppressor gene in human nonsmall-cell lung cancer. Nat Genet 27:427-430.

Latefi NS, Colman DR (2007) The CNS synapse revisited: gaps, adhesive welds, and borders. Neurochem Res 32:303-310.

Levinson JN, Chery N, Huang K, Wong TP, Gerrow K, Kang R, Prange O, Wang YT, El-Husseini A (2005) Neuroligins mediate excitatory and inhibitory synapse formation: involvement of PSD-95 and neurexin-1beta in neuroligin-induced synaptic specificity. J Biol Chem 280:17312-17319.

Lucic V, Yang T, Schweikert G, Forster F, Baumeister W (2005) Morphological characterization of molecular complexes present in the synaptic cleft. Structure (Camb) 13:423-434.

Matteoli M, Takei K, Perin MS, Südhof TC, De Camilli P (1992) Exoendocytotic recycling of synaptic vesicles in developing processes of cultured hippocampal neurons. J Cell Biol 117:849-861.

Maurel P, Einheber S, Galinska J, Thaker P, Lam I, Rubin MB, Scherer SS, Murakami Y, Gutmann DH, Salzer JL (2007) Nectin-like proteins mediate axon Schwann cell interactions along the internode and are essential for myelination. J Cell Biol 178:861-874.

Mayford M, Barzilai A, Keller F, Schacher S, Kandel ER (1992) Modulation of an NCAM-related adhesion molecule with long-term synaptic plasticity in Aplysia. Science 256:638-644.

Missler M, Zhang WQ, Rohlmann A, Kattenstroth G, Hammer RE, Gottmann K, Südhof TC (2003) alpha-Neurexins couple Ca2+ channels to synaptic vesicle exocytosis. Nature 423:939-948.

Mullen RJ, Buck CR, Smith AM (1992) NeuN, a neuronal specific nuclear protein in vertebrates. Development 116:201-211.

Murakami Y (2005) Involvement of a cell adhesion molecule, TSLC1/ IGSF4, in human oncogenesis. Cancer Sci 96:543-552.

Niwa H, Yamamura K, Miyazaki J (1991) Efficient selection for highexpression transfectants with a novel eukaryotic vector. Gene 108:193-199.

Sara Y, Biederer T, Atasoy D, Chubykin A, Mozhayeva MG, Südhof TC, Kavalali ET (2005) Selective capability of SynCAM and neuroligin for functional synapse assembly. J Neurosci 25:260-270.

Scheiffele P (2003) Cell-cell signaling during synapse formation in the CNS. Annu Rev Neurosci 26:485-508.

Scheiffele P, Fan J, Choih J, Fetter R, Serafini T (2000) Neuroligin expressed in nonneuronal cells triggers presynaptic development in contacting axons. Cell 101:657-669.
Schikorski T, Stevens CF (1997) Quantitative ultrastructural analysis of hippocampal excitatory synapses. J Neurosci 17:5858-5867.

Shen K, Bargmann CI (2003) The immunoglobulin superfamily protein SYG-1 determines the location of specific synapses in C. elegans. Cell 112:619-630.

Shen K, Fetter RD, Bargmann CI (2004) Synaptic specificity is generated by the synaptic guidepost protein SYG-2 and its receptor, SYG-1. Cell 116:869-881.

Shingai T, Ikeda W, Kakunaga S, Morimoto K, Takekuni K, Itoh S, Satoh K, Takeuchi M, Imai T, Monden M, Takai Y (2003) Implications of nectinlike molecule-2/IGSF4/RA175/SgIGSF/TSLC1/SynCAM1 in cell-cell adhesion and transmembrane protein localization in epithelial cells. J Biol Chem 278:35421-35427.

Song JY, Ichtchenko K, Südhof TC, Brose N (1999) Neuroligin 1 is a postsynaptic cell-adhesion molecule of excitatory synapses. Proc Natl Acad Sci USA 96:1100-1105.

Spiegel I, Adamsky K, Eshed Y, Milo R, Sabanay H, Sarig-Nadir O, Horresh I, Scherer SS, Rasband MN, Peles E (2007) A central role for Necl4 (SynCAM4) in Schwann cell-axon interaction and myelination. Nat Neurosci 10:861-869.

Sugita S, Sudhof TC (2000) Specificity of Ca2+-dependent protein interactions mediated by the $\mathrm{C} 2 \mathrm{~A}$ domains of synaptotagmins. Biochemistry 39:2940-2949.

Sugita S, Saito F, Tang J, Satz J, Campbell K, Sudhof TC (2001) A stoichiometric complex of neurexins and dystroglycan in brain. J Cell Biol 154:435-445.

Takamori S, Holt M, Stenius K, Lemke EA, Gronborg M, Riedel D, Urlaub H, Schenck S, Brugger B, Ringler P, Muller SA, Rammner B, Grater F, Hub JS, De Groot BL, Mieskes G, Moriyama Y, Klingauf J, Grubmuller H, Heuser J, Wieland F, Jahn R (2006) Molecular anatomy of a trafficking organelle. Cell 127:831-846.

Takeichi M (2007) The cadherin superfamily in neuronal connections and interactions. Nat Rev Neurosci 8:11-20.

Tanaka H, Shan W, Phillips GR, Arndt K, Bozdagi O, Shapiro L, Huntley GW, Benson DL, Colman DR (2000) Molecular modification of N-cadherin in response to synaptic activity. Neuron 25:93-107.

Taniguchi H, Gollan L, Scholl FG, Mahadomrongkul V, Dobler E, Limthong N, Peck M, Aoki C, Scheiffele P (2007) Silencing of neuroligin function by postsynaptic neurexins. J Neurosci 27:2815-2824.

Uchida N, Honjo Y, Johnson KR, Wheelock MJ, Takeichi M (1996) The catenin/cadherin adhesion system is localized in synaptic junctions bordering transmitter release zones. J Cell Biol 135:767-779.

Urase K, Soyama A, Fujita E, Momoi T (2001) Expression of RAI75 mRNA, a new member of the immunoglobulin superfamily, in developing mouse brain. NeuroReport 12:3217-3221.

Ushkaryov YA, Südhof TC (1993) Neurexin III alpha: extensive alternative splicing generates membrane-bound and soluble forms. Proc Natl Acad Sci USA 90:6410-6414.

Ushkaryov YA, Petrenko AG, Geppert M, Südhof TC (1992) Neurexins: synaptic cell surface proteins related to the alpha- latrotoxin receptor and laminin. Science 257:50-56.

Varoqueaux F, Aramuni G, Rawson RL, Mohrmann R, Missler M, Gottmann K, Zhang W, Sudhof TC, Brose N (2006) Neuroligins determine synapse maturation and function. Neuron 51:741-754.

Waites CL, Marie Craig A, Garner CC (2005) Mechanisms of vertebrate synaptogenesis. Annu Rev Neurosci 28:251-274.

Wang CY, Chang K, Petralia RS, Wang YX, Seabold GK, Wenthold RJ (2006) A novel family of adhesion-like molecules that interacts with the NMDA receptor. J Neurosci 26:2174-2183.

Yamagata M, Sanes JR, Weiner JA (2003) Synaptic adhesion molecules. Curr Opin Cell Biol 15:621-632. 